\title{
Orbifold points on Teichmüller curves and Jacobians with complex multiplication
}

by

Ronen E. Mukamel

Submitted to the Department of Mathematics in partial fulfillment of the requirements for the degree of

Doctor of Philosophy

at the

\section{MASSACHUSETTS INSTITUTE OF TECHNOLOGY}

June 2011

(C) Ronen E. Mukamel, MMXI. All rights reserved.

The author hereby grants to MIT permission to reproduce and distribute publicly paper and electronic copies of this thesis document in whole or in part.

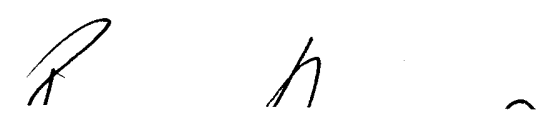

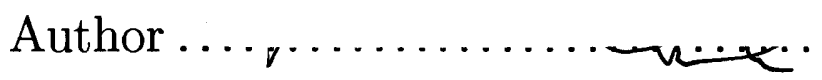

Department of Mathematics

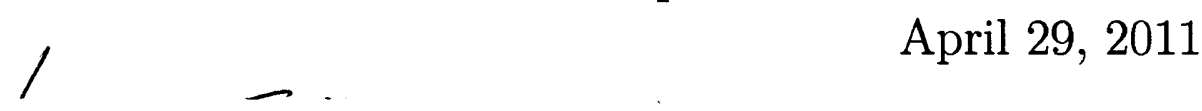

Certified by

Curtis T. McMullen

Professor

Thesis Supervisor

Accepted by

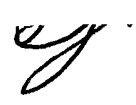

Bjorn Poonen

Chairman, Department Committee on Graduate Theses 


\title{
Orbifold points on Teichmüller curves and Jacobians with complex multiplication
}

\author{
by \\ Ronen E. Mukamel \\ Submitted to the Department of Mathematics \\ on April 29, 2011, in partial fulfillment of the \\ requirements for the degree of \\ Doctor of Philosophy
}

\begin{abstract}
For each integer $D \geq 5$ with $D \equiv 0$ or $1 \bmod 4$, the Weierstrass curve $W_{D}$ is an algebraic curve and a finite volume hyperbolic orbifold which admits an algebraic and isometric immersion into the moduli space of genus two Riemann surfaces. The Weierstrass curves are the main examples of Teichmüller curves in genus two.

The primary goal of this thesis is to determine the number and type of orbifold points on each component of $W_{D}$. Our enumeration of the orbifold points, together with [Ba] and [Mc3], completes the determination of the homeomorphism type of $W_{D}$ and gives a formula for the genus of its components. We use our formula to give bounds on the genus of $W_{D}$ and determine the Weierstrass curves of genus zero. We will also give several explicit descriptions of each surface labeled by an orbifold point on $W_{D}$.
\end{abstract}

Thesis Supervisor: Curtis T. McMullen

Title: Professor 



\section{Acknowledgments}

First and foremost, I would like to thank my advisor, Curt McMullen, for his help and guidance during the writing of this thesis. He has been mathematically very generous to me, and I deeply appreciate all that he has taught me. I would also like to thank my other readers-Dick Gross, Abhinav Kumar, and Bjorn Poonen-as well as Anatoly Preygel and Vaibhav Gadre for their useful comments and suggestions. I have had many other inspiring mathematics teachers over the years-including Fred Cohen, Joe Harris, Peter Kronheimer and Arnold Ross-and I would like to thank them as well. I would also like to thank my family-Amelia, Eran, Dana and Shaulfor their continued love and support. Finally, I would like to thank my dear friends who have made the years I have spent graduate school very special. In no particular alphabetical order they include: Ailsa, Alex, Amelia, Bea, Christa, Dustin, Ethan, Griselda, Helen, Liz, Pat, Pat, Jacob, Jacob, Jim, Nick, Olga, Toly and Won. 


\section{Contents}

1 Introduction $\quad 9$

2 Background 21

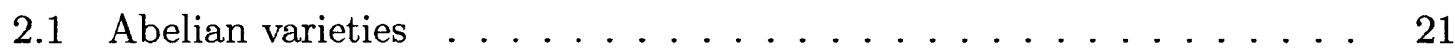

2.2 Riemann surfaces, Jacobians and Automorphisms . . . . . . . . 28

2.3 The Weierstrass curve ................... 34

3 Orbifold points on Hilbert modular surfaces $\quad 39$

4 The $D_{8}$-family $\quad 45$

$\begin{array}{lll}5 & \text { Endomorphisms } & \mathbf{5 7}\end{array}$

$\begin{array}{lll}6 & \text { Spin } & 63\end{array}$

$\begin{array}{lll}7 & \text { Genus } & 67\end{array}$

$\begin{array}{ll}\text { A The } D_{12} \text {-family } & 75\end{array}$

A.1 The $D_{12}$-family . . . . . . . . . . . . . . . 75

A.2 Homeomorphism type of $W_{D} \ldots \ldots \ldots$. . . . . . . 79 


\section{Chapter 1}

\section{Introduction}

Let $\mathcal{M}_{g}$ be the moduli space of genus $g$ Riemann surfaces. The space $\mathcal{M}_{g}$ can be viewed as both a complex orbifold and an algebraic variety and carries a complete Teichmüller metric. A Teichmüller curve is an algebraic and isometric immersion of a finite volume hyperbolic Riemann surface:

$$
f: C=\mathbb{H} / \Gamma \rightarrow \mathcal{M}_{g}
$$

The modular curve $\mathcal{M}_{1}$ is the first example of a Teichmüller curve. Other examples emerge from the study of polygonal billiards [Ve, MT] and square-tiled surfaces. While the Teichmüller curves in $\mathcal{M}_{2}$ have been classified [Mc5], much less is known about Teichmüller curves in $\mathcal{M}_{g}$ for $g>2$ [BaM, BM2].

For each integer $D \geq 5$ with $D \equiv 0$ or $1 \bmod 4$, the Weierstrass curve $W_{D}$ is the moduli space of Riemann surfaces whose Jacobians have real multiplication by the quadratic order $\mathcal{O}_{D}=\mathbb{Z}\left[\frac{D+\sqrt{D}}{2}\right]$ stabilizing a holomorphic one form with double zero up to scale. The curve $W_{D}$ is a finite volume hyperbolic orbifold and the natural immersion:

$$
W_{D} \rightarrow \mathcal{M}_{2}
$$

is algebraic and isometric and has degree one onto its image [Ca, Mc1]. The curve $W_{D}$ is a Teichmüller curve unless $D>9$ with $D \equiv 1 \bmod 8$ in which case $W_{D}=W_{D}^{0} \sqcup W_{D}^{1}$ is a disjoint union of two Teichmüller curves distinguished by a spin invariant in 


\begin{tabular}{|c|c|}
\hline$D \bmod 16$ & $e_{2}\left(W_{D}\right)$ \\
\hline $1,5,9$, or 13 & $\frac{1}{2} \widetilde{h}(-4 D)$ \\
0 & $\frac{1}{2}(\widetilde{h}(-D)+2 \widetilde{h}(-D / 4))$ \\
4 & 0 \\
8 & $\frac{1}{2} \widetilde{h}(-D)$ \\
12 & $\frac{1}{2}(\widetilde{h}(-D)+3 \widetilde{h}(-D / 4))$ \\
\hline
\end{tabular}

Table 1.1: For $D>8$, the number of orbifold points of order two on $W_{D}$ is given by a weighted sum of class numbers. The function $\widetilde{h}(-D)$ is defined below.

$\mathbb{Z} / 2 \mathbb{Z}$ [Mc3]. A major challenge is to describe $W_{D}$ as an algebraic curve and as a hyperbolic orbifold. To date, this has been accomplished only for certain small $D$ [BM1, Mc1, Lo].

The purpose of this thesis is to study the orbifold points on $W_{D}$. Such points label surfaces with automorphisms commuting with $\mathcal{O}_{D}$. The first two Weierstrass curves $W_{5}$ and $W_{8}$ were studied by Veech $[\mathrm{Ve}]$ and are isomorphic to the $(2,5, \infty)$ - and $(4, \infty, \infty)$-orbifolds. The surfaces with automorphisms labeled by the three orbifold points are drawn in Figure 1-1.

Our primary goal is to give a formula for the number and type of orbifold points on $W_{D}$. Together with [Mc3] and [Ba], our formula completes the determination of the homeomorphism type of $W_{D}$ and gives a formula for the genus of $W_{D}$. We will use our formula to give bounds for the genera of $W_{D}$ and $W_{D}^{\epsilon}$ and list the components of $\bigcup_{D} W_{D}$ of genus zero. We will also give several explicit descriptions of the surfaces labeled by orbifold points on $W_{D}$, giving the first examples of algebraic curves labeled by points of $W_{D}$ for most $D$.

Main results. Our main theorem determines the number and type of orbifold points on $W_{D}$ :

Theorem 1.1. For $D>8$, the orbifold points on $W_{D}$ all have order two, and the number of such points $e_{2}\left(W_{D}\right)$ is the weighted sum of class numbers of imaginary quadratic orders shown in Table 1.1. 
We also give a formula for the number of orbifold points on each spin component:

Theorem 1.2. Fix $D \geq 9$ with $D \equiv 1 \bmod 8$. If $D=f^{2}$ is a perfect square, then all of the orbifold points on $W_{D}$ lie on the component with spin $(f+1) / 2 \bmod 2$ :

$$
e_{2}\left(W_{D}^{(f+1) / 2}\right)=\frac{1}{2} \widetilde{h}(-4 D) \text { and } e_{2}\left(W_{D}^{(f-1) / 2}\right)=0
$$

Otherwise, $e_{2}\left(W_{D}^{0}\right)=e_{2}\left(W_{D}^{1}\right)=\frac{1}{4} \widetilde{h}(-4 D)$.

When $D$ is not a square and $W_{D}$ is reducible, the spin components of $W_{D}$ have algebraic models defined over $\mathbb{Q}(\sqrt{D})$ and are Galois conjugate [BM1]. Theorem 1.2 confirms that the spin components have the same number and type of orbifold points.

The class number $h(-D)$ is the order of the ideal class group $H(-D)$ for $\mathcal{O}_{-D}$ and counts the number of elliptic curves with complex multiplication by $\mathcal{O}_{-D}$ up to isomorphism. The weighted class number

$$
\widetilde{h}(-D)=2 h(-D) /\left|\mathcal{O}_{-D}^{\times}\right|
$$

appearing in Table 1.1 is the number of elliptic curves with complex multiplication weighted by their orbifold order in $\mathcal{M}_{1}$. Note that $\widetilde{h}(-D)=h(-D)$ unless $D=3$ or 4. When $D$ is odd, the orbifold points on $W_{D}$ are labeled by elements of the group $H(-4 D) /[P]$ where $[P]$ is the ideal class in $\mathcal{O}_{-4 D}$ representing the prime ideal with norm two.

The orbifold Euler characteristics of $W_{D}$ and $W_{D}^{\epsilon}$ were computed in [Ba] and the cusps on $W_{D}$ were enumerated and sorted by component in [Mc3]. Theorems 1.1 and 1.2 complete the determination of the homeomorphism type of $W_{D}$ and give a formula for the genera of $W_{D}$ and its components.

Corollary 1.3. For any $\epsilon>0$, there are constants $C_{\epsilon}$ and $N_{\epsilon}$ such that:

$$
C_{\epsilon} D^{3 / 2+\epsilon}>g(V)>D^{3 / 2} / 650
$$

whenever $V$ is a component of $W_{D}$ and $D \geq N_{\epsilon}$. 

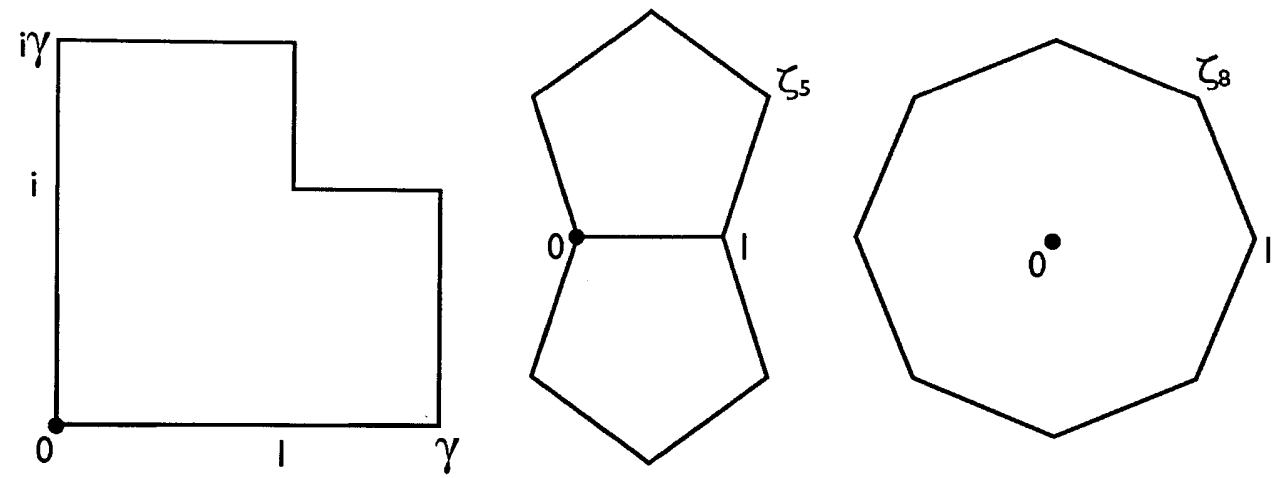

Figure 1-1: The first two Weierstrass curves $W_{5}$ and $W_{8}$ are isomorphic to the $(2,5, \infty)$ and $(4, \infty, \infty)$-orbifolds. The point of order two is related to billiards on the $L$-shaped table (left) corresponding to the golden mean $\gamma=\frac{1+\sqrt{5}}{2}$. The points of order five (center) and four (right) are related to billiards on the regular pentagon and octagon.

Modular curves of genus zero play an important role in number theory [Ti]. We also determine the components of Weierstrass curves of genus zero.

Corollary 1.4. The genus zero components of $\bigcup_{D} W_{D}$ are the 23 components of $\bigcup_{D \leq 41} W_{D}$ and the curves $W_{49}^{0}, W_{49}^{1}$ and $W_{81}^{1}$.

We include a table listing the homeomorphism type of $W_{D}$ for $D<250$ in $\S A .2$.

Orbifold points on Hilbert modular surfaces. Theorem 1.1 is closely related to the classification of orbifold points on Hilbert modular surfaces we prove in $\S 3$. The Hilbert modular surface $X_{D}$ is the moduli space of principally polarized abelian varieties with real multiplication by $\mathcal{O}_{D}$. The period map sending a Riemann surface to its Jacobian embeds $W_{D}$ in $X_{D}$.

Central to the story of the orbifold points on $X_{D}$ and $W_{D}$ are the moduli spaces $\mathcal{M}_{2}\left(D_{8}\right)$ and $\mathcal{M}_{2}\left(D_{12}\right)$ of genus two surfaces with actions of the dihedral groups of orders 8 and 12:

$$
D_{8}=\left\langle r, J: r^{2}=(J r)^{2}=J^{4}=1\right\rangle \text { and } D_{12}=\left\langle r, Z: r^{2}=(Z r)^{2}=Z^{6}=1\right\rangle \text {. }
$$

The surfaces in $\mathcal{M}_{2}\left(D_{8}\right)$ (respectively $\mathcal{M}_{2}\left(D_{12}\right)$ ) whose Jacobians have complex mul- 
tiplication have real multiplication commuting with $J$ (respectively $Z$ ). The complex multiplication points on $\mathcal{M}_{2}\left(D_{8}\right)$ and $\mathcal{M}_{2}\left(D_{12}\right)$ give most of the orbifold points on $\bigcup_{D} X_{D}$ :

Theorem 1.5. The orbifold points on $\bigcup_{D} X_{D}$ which are not products of elliptic curves are the two points of order five on $X_{5}$ and the complex multiplication points on $\mathcal{M}_{2}\left(D_{8}\right)$ and $\mathcal{M}_{2}\left(D_{12}\right)$.

Since the $Z$-eigenforms on $D_{12}$-surfaces have simple zeros and the $J$-eigenforms on $D_{8}$-surfaces have double zeros (cf. Proposition 3.2), we have:

Corollary 1.6. The orbifold points on $\bigcup_{D} W_{D}$ are the point of order five on $W_{5}$ and the complex multiplication points on $\mathcal{M}_{2}\left(D_{8}\right)$.

Corollary 1.6 explains the appearance of class numbers in the formula for $e_{2}\left(W_{D}\right)$. The involutions $r$ and $J r$ on a $D_{8}$-surface $X$ have genus one quotients $X / r$ and $X / J r$ whose Jacobians are related by a degree two isogeny and the family $\mathcal{M}_{2}\left(D_{8}\right)$ is birational to the modular curve $Y_{0}(2)$. The Jacobian $\operatorname{Jac}(X)$ has complex multiplication by an order in $\mathbb{Q}(\sqrt{D}, i)$ if and only if $\operatorname{Jac}(X / r)$ has complex multiplication by an order in $\mathbb{Q}(\sqrt{-D})$. The formula for $e_{2}\left(W_{D}\right)$ follows by sorting the $3 \widetilde{h}(-D)$ surfaces with $D_{8}$-action covering elliptic curves with complex multiplication by $\mathcal{O}_{-D}$ by their orders for real multiplication.

The product locus $P_{D}$. A recurring theme in the study of the Weierstrass curves is the close relationship between $W_{D}$ and the product locus $P_{D} \subset X_{D}$. The product locus $P_{D}$ consists of products of elliptic curves with real multiplication by $\mathcal{O}_{D}$.

The cusps on $W_{D}$ were first enumerated and sorted by spin in [Mc3] and, for non-square $D$, are in bijection with the cusps on $P_{D}$ (cf. $\S 7$ ). The Hilbert modular surface $X_{D}$ has a meromorphic modular form with a simple pole along $P_{D}$ and a simple zero along $W_{D}$. This modular form can be used to give a formula for the Euler characteristic of $W_{D}$ and, for non-square $D$, the Euler characteristics of $W_{D}, X_{D}$ and $P_{D}$ satisfy ([Ba], Cor. 10.4):

$$
\chi\left(W_{D}\right)=\chi\left(P_{D}\right)-2 \chi\left(X_{D}\right)
$$


Our classification of the orbifold points on $X_{D}$ and $W_{D}$ in Theorem 1.5 and Corollary 1.6 show that all of the orbifold points of order two on $X_{D}$ lie on $W_{D}$ or $P_{D}$.

Theorem 1.7. For non-square $D$, the homeomorphism type of $W_{D}$ is determined by the homeomorphism types of $X_{D}$ and $P_{D}$ and $D \bmod 8$.

The $D_{8}$-family. A secondary goal of our analysis is to give several explicit descriptions of $D_{8}$-surfaces and to characterize those with complex multiplication. We will outline a similar discussion for $\mathcal{M}_{2}\left(D_{12}\right)$ in $\S$ A.1. For a genus two surface $X \in \mathcal{M}_{2}$, the following are equivalent:

1. Automorphisms. The automorphism group $\operatorname{Aut}(X)$ admits an injective homomorphism $\rho: D_{8} \rightarrow \operatorname{Aut}(X)$.

2. Algebraic curves. The field of meromorphic functions $\mathbb{C}(X)$ is isomorphic to:

$$
K_{a}=\mathbb{C}(z, x) \text { with } z^{2}=\left(x^{2}-1\right)\left(x^{4}-a x^{2}+1\right),
$$

for some $a \in \mathbb{C} \backslash\{ \pm 2\}$.

3. Jacobians. There is a number $\tau \in \mathbb{H}$ such that the $\operatorname{Jacobian} \operatorname{Jac}(X)$ is isomorphic to the principally polarized abelian variety:

$$
A_{\tau}=\mathbb{C}^{2} / \Lambda_{\tau}
$$

where $\Lambda_{\tau}=\mathbb{Z}\left\langle\left(\begin{array}{c}\tau \\ \tau+1\end{array}\right),\left(\begin{array}{c}\tau \\ -\tau-1\end{array}\right),\left({ }_{\tau}^{\tau+1}\right),\left(\begin{array}{c}\tau+1 \\ -\tau\end{array}\right)\right\rangle$ and $A_{\tau}$ is polarized by the symplectic form $\left\langle\left(\begin{array}{c}a \\ b\end{array}\right),\left(\begin{array}{c}c \\ d\end{array}\right)\right\rangle=\frac{\operatorname{Im}(a \bar{c}+b \bar{d})}{2 \operatorname{Im}(\tau)}$.

4. Pinwheels. The surface $X$ is isomorphic to the surface $X_{\tau}$ obtained from the polygonal pinwheel $P_{\tau}$ (Figure 1-2) for some $\tau$ in the domain:

$$
U=\left\{\tau \in \mathbb{H}: \tau \neq \frac{ \pm 1+i}{2},|\tau|^{2} \geq \frac{1}{2} \text { and }|\operatorname{Re} \tau| \leq \frac{1}{2}\right\}
$$




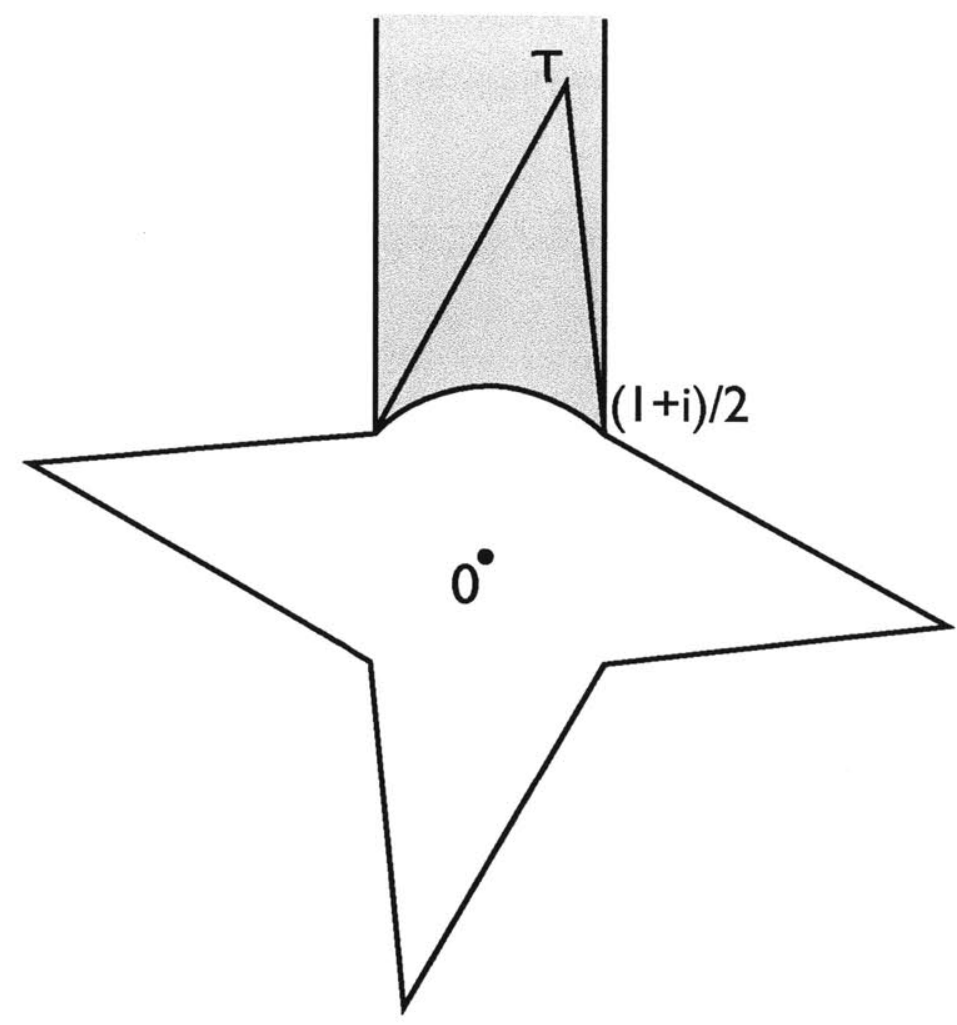

Figure 1-2: For $\tau$ in the shaded domain $U$, the pinwheel $P_{\tau}$ has vertices at $z=\frac{1 \pm i}{2}, \frac{-1 \pm i}{2}$, $\pm \tau$, and $\pm i \tau$. Gluing together opposite sides on $P_{\tau}$ by translation gives a genus two surface admitting an action of $D_{8}$. The one form induced by $d z$ is a $J$-eigenform and has a double zero.

5. Parallelograms. The surface $X$ is isomorphic to the surface $Y_{w}$ obtained from the parallelogram $Q_{w}$ (Figure 1-3) for some $w$ in the domain:

$$
V=\{w \in \mathbb{H}: 0 \leq \operatorname{Re} w \leq 2 \text { and }|w-1| \geq 1\}
$$

It is straightforward to identify the action of $D_{8}$ in each of the descriptions above. The field $K_{a}$ has automorphisms $r(z, x)=(z,-x)$ and $J(z, x)=\left(i z / x^{3}, 1 / x\right)$. The linear transformations $r=\left(\begin{array}{cc}1 & 0 \\ 0 & -1\end{array}\right)$ and $J=\left(\begin{array}{cc}0 & 1 \\ -1 & 0\end{array}\right)$ preserve the polarized lattice $\Lambda_{\tau}$. The action of $D_{8}$ on the surface $Y_{w}$ is identified in Figure 1-3. The surface $X_{\tau}$ satisfies $\mathbb{C}\left(X_{\tau}\right) \cong K_{a}$ for some $a$ because the order four automorphism obtained by rotating $P_{\tau}$ acts as a product of two disjoint transpositions on the Weierstrass points of $X_{\tau}$ (cf. Propositions 3.2 and 3.3). 
The function relating the number $\tau$ determining the polygon $P_{\tau}$ and abelian variety $A_{\tau}$ to the number $a$ determining the field $K_{a}$ is the modular function:

$$
a(\tau)=-2+\frac{1}{\lambda(\tau) \lambda(\tau+1)}
$$

The function $\lambda(\tau)$ is modular for the group $\Gamma(2)=\operatorname{ker}\left(\mathrm{SL}_{2}(\mathbb{Z}) \rightarrow \mathrm{SL}_{2}(\mathbb{Z} / 2 \mathbb{Z})\right)$ and descends to the isomorphism $\lambda: \mathbb{H} / \Gamma(2) \stackrel{\sim}{\rightarrow} \mathbb{C} \backslash\{0,1\}$ sending the cusps $\Gamma(2) \cdot 0$, $\Gamma(2) \cdot 1$ and $\Gamma \cdot \infty$ to 0,1 and $\infty$ respectively. The function relating $\tau$ and $w$ is the Riemann mapping $w: U \rightarrow V$ whose extension to $\partial U$ has $w((-1+i) / 2)=0$, $w((1+i) / 2)=2$ and $w(\infty)=\infty$. In Sections 4 and 5 we will prove:

Theorem 1.8. Fix $\tau \in U$. The surface $X_{\tau}$ obtained from the polygon $P_{\tau}$ admits a faithful $D_{8}$-action, is isomorphic to the surface obtained from the parallelogram $Q_{w(\tau)}$ and satisfies:

$$
\operatorname{Jac}\left(X_{\tau}\right) \cong A_{\tau} \text { and } \mathbb{C}\left(X_{\tau}\right) \cong K_{a(\tau)}
$$

The Jacobian $\operatorname{Jac}\left(X_{\tau}\right)$ has complex multiplication if and only if $\tau$ is imaginary quadratic.

Teichmüller and Shimura curves. There are very few examples of Teichmüller curves parametrizing surfaces whose Jacobians lie on a Shimura curve [Mo]. The families $\mathcal{M}_{2}\left(D_{8}\right)$ and $\mathcal{M}_{2}\left(D_{12}\right)$ are examples of Teichmüller-Shimura curves and are related to the known examples by a branched covering construction.

The families $\mathcal{M}_{2}\left(D_{8}\right)$ and $\mathcal{M}_{2}\left(D_{12}\right)$ are Teichmüller curves arising from squaretiled surfaces (see Figures 1-3 and A-2). The Jacobians of $D_{8}$ - and $D_{12}$-surfaces lie on different components of the transverse self-intersection of the immersion of $X_{4}$ into the moduli space of principally polarized abelian varieties. The Jacobians of $D_{8^{-}}$and $D_{12}$-surfaces admit proper actions of the involutive rings

$$
R_{D_{8}}=\mathbb{Z}\left[D_{8}\right] /\left(J^{2}+1\right) \text { and } R_{D_{12}}=\mathbb{Z}\left[D_{12}\right] /\left(Z^{3}+1\right)
$$

such that the actions of $r$ and $J r$ in $D_{8}$ and $r$ and $Z r$ in $D_{12}$ are self-adjoint. The Weierstrass curve $W_{D}$, by contrast, is not a Shimura curve ([Mc1], Cor. 10.2) be- 


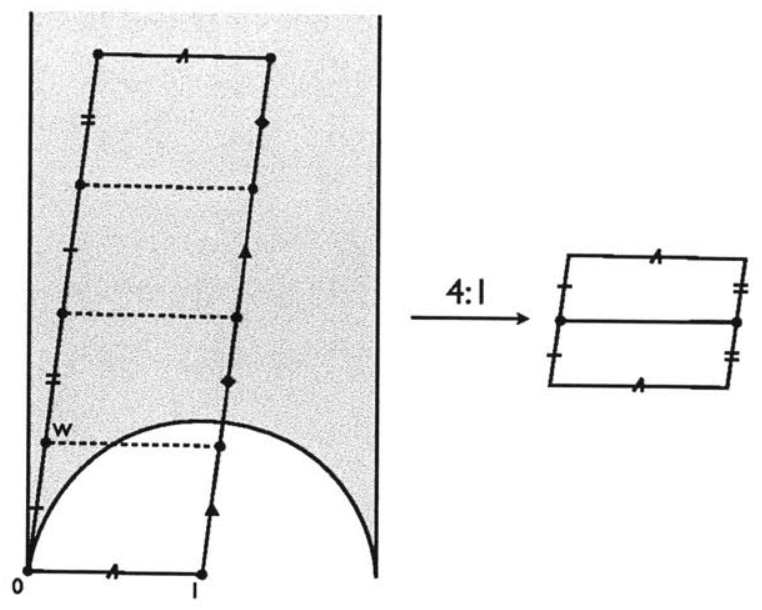

Figure 1-3: For $w$ in the shaded domain $V$, the parallelogram $Q_{w}$ has vertices $\{0,1,4 w, 4 w+1\}$. Gluing the marked segments together as indicated gives a genus two surface $Y_{w}$ with a degree four cyclic covering map to the double of a parallelogram. The surface $Y_{w}$ has an action of $D_{8}$ generated by the Deck transformation and the involution covered by $z \mapsto-z+2 w+1$. The quadratic differential $q$ on $Y_{w}$ induced by $d z^{2}$ has two double zeros and is a product of $J$-eigenforms.

cause of the condition on the $\mathcal{O}_{D}$-eigenform. The abelian varieties in $X_{D}$ which are Jacobians of surfaces in $W_{D}$ cannot be distinguished by their endomorphism rings.

Algebraic models for $D_{8}$-surfaces with complex multiplication. The function $a(\tau)$ is a transcendental function of $\tau$ and modular for the group $\Gamma_{0}(2)=$ $\left\langle\Gamma(2),\left(\begin{array}{ll}1 & 1 \\ 0 & 1\end{array}\right)\right\rangle$. Setting $q=e^{2 \pi i \tau}$, we have:

$$
a(\tau)=-2-256 q-6144 q^{2}-76800 q^{3}-671744 q^{4}+\ldots
$$

Usually, at most one of $\tau$ and $a(\tau)$ is algebraic. When $\tau$ is imaginary quadratic, the number $a(\tau)$ generates a finite abelian extension of $\mathbb{Q}(\tau)$. The $D_{8}$-surfaces with complex multiplication give the first explicit examples of algebraic curves labeled by points on $W_{D}$ for most $D$.

For instance, when $\tau=\frac{1+\sqrt{-19}}{2}, X_{\tau}$ is labeled by an orbifold point on $W_{76}$ and $a(\tau)$ generates a degree three abelian extension of $\mathbb{Q}(\sqrt{-19})$. Computer experiment 
indicates that the number $a(\tau)=-1.999710899 \ldots$ is the unique real root of:

$$
x^{3}+3 x^{2}+3459 x+6913=0 .
$$

Similarly, the point of order two on $W_{5}$ is obtained from the polygon $P_{\frac{1}{2}(1+\sqrt{-5})}$ and $a\left(\frac{1}{2}(1+\sqrt{-5})\right)=34-16 \sqrt{5}$. The point of order four on $W_{8}$ is obtained from the polygon $P_{\sqrt{-2} / 2}$ and $a(\sqrt{-2} / 2)=-6$.

Outline. We conclude this Introduction with an outline of the proofs of our main results.

1. An automorphism $\phi$ on a surface $X \in \mathcal{M}_{2}$ induces a permutation $\sigma_{\phi}$ on the Weierstrass points. A brief analysis of the possibilities for $\sigma_{\phi}$ shows that the most of the orbifold points on $\bigcup_{D} X_{D}$ which are not products of elliptic curves lie on $\mathcal{M}_{2}\left(D_{8}\right)$ and $\mathcal{M}_{2}\left(D_{12}\right)$ and that most of the orbifold points on $\bigcup_{D} W_{D}$ lie on $\mathcal{M}_{2}\left(D_{8}\right)(\S 3)$.

2. In $\S 4$ we study the $D_{8}$-family. For a genus two surface $X$ with faithful $D_{8}$-action $\rho$, the correspondence $X \rightarrow X / r \times X / J r$ induces a degree two isogeny between elliptic curves:

$$
c_{\rho}: \operatorname{Jac}(X / r) \rightarrow \operatorname{Jac}(X / J r) .
$$

We compute $\mathbb{C}(X)$ and $\operatorname{Jac}(X)$ in terms of the isogeny $c_{\rho}$ and show that the map $(X, \rho) \mapsto c_{\rho}$ embeds $\mathcal{M}_{2}\left(D_{8}\right)$ as the complement the degree two endomorphism of the square torus in the modular curve $Y_{0}(2)$ parametrizing degree two isogenies between elliptic curves. We then prove Theorem 1.8 by computing the isogenies associated to the $D_{8}$-actions on the surfaces $X_{\tau}$ and $Y_{w(\tau)}$. The outer automorphism $\sigma$ of $D_{8}$ acts on $\mathcal{M}_{2}\left(D_{8}\right)$ and induces the Atkin-Lehner involution on $Y_{0}(2)$ sending an isogeny to its dual. Note that the domain $U$ for pinwheels is a fundamental domain for the group $\left\langle\Gamma_{0}(2), \sigma=\left(\begin{array}{cc}0 & 1 / \sqrt{2} \\ \sqrt{2} & 0\end{array}\right)\right\rangle$. The family $\mathcal{M}_{2}\left(D_{12}\right)$ admits a similar analysis, which we outline in $\S \mathrm{A} .1$.

3. Let $(X, \rho)$ be a $D_{8}$-surface and let $E=\operatorname{Jac}(X / r)$. In $\S 5$ we use the isogeny 
$\operatorname{Jac}(X) \rightarrow E \times E$ induced by the map $X \rightarrow X / r \times X / \eta r$ to embed $\operatorname{End}(\operatorname{Jac}(X))$ as an order in $M_{2}(\operatorname{End}(E) \otimes \mathbb{Q})$. It follows that $\operatorname{Jac}(X)$ has complex multiplication if and only if $E$ has complex multiplication and that the complex multiplication points on $\mathcal{M}_{2}\left(D_{8}\right)$ give orbifold points on Weierstrass curves. We then sort the $3 \widetilde{h}(-D)$ surfaces with $D_{8}$-action covering elliptic curves with complex multiplication by $\mathcal{O}_{-D}$ by their orders for real multiplication commuting with $J$ giving the formula for $e_{2}\left(W_{D}\right)$ in Theorem 1.1.

4. In $\S 6$ we sort the orbifold points on $W_{D}$ by spin component when $D \equiv 1 \bmod 8$. For such discriminants, the orbifold points on $W_{D}$ correspond to ideal classes for $\mathcal{O}_{-4 D}$. These form a group $H(-4 D)$ and there is a spin homomorphism:

$$
\epsilon_{0}: H(-4 D) \rightarrow \mathbb{Z} / 2 \mathbb{Z}
$$

Fix a proper ideal $I$ for $\mathcal{O}_{-4 D}$ and let $(X, \rho)$ be the $D_{8}$-surface with $X / r \cong \mathbb{C} / I$ and with real multiplication by $\mathcal{O}_{D}$. The spin-invariant for the corresponding orbifold point on $W_{D}$ is given by:

$$
\epsilon=\frac{f+1}{2}+\epsilon_{0}(I) \bmod 2
$$

where $f$ is the conductor of $\mathcal{O}_{D}$, i.e. the index of $\mathcal{O}_{D}$ in the maximal order of $\mathbb{Q}(\sqrt{D})$. The spin homomorphism is the zero map iff $D$ is a square giving the formula in Theorem 1.2.

5. In $\S 7$ we use the formula for the number and type of orbifold points on $W_{D}$ to give bounds on the genera of the components of $W_{D}$.

Open problems. While the homeomorphism type of $W_{D}$ is now understood, describing the components of $W_{D}$ as Riemann surfaces remains a challenge.

Problem 1. Describe $W_{D}$ as a hyperbolic orbifold and as an algebraic curve.

Our analysis of the orbifold points on $W_{D}$ have given explicit descriptions of some complex multiplication points on $W_{D}$. By the André-Oort conjecture [KY], there are 
only finitely many complex multiplication points on $W_{D}$ and it would be interesting to find them.

Problem 2. Describe the complex multiplication points on $W_{D}$.

The complex multiplication points on $\mathcal{M}_{2}\left(D_{8}\right)$ generate Teichmüller curves and the complex multiplication points on $\mathcal{M}_{2}\left(D_{12}\right)$ generate complex geodesics with infinitely generated fundamental group. It would be interesting to find other examples of Shimura varieties whose complex multiplication points lie on interesting complex geodesics.

Problem 3. Find other Shimura varieties whose complex multiplication points generate Teichmüller curves.

The divisors supported at cusps on modular curves generate a finite subgroup of the associated Jacobian [Ma]. It would be interesting to know if the same is true for Teichmüller curves. The first Weierstrass curve with genus one is $W_{\mathbf{4 4}}$.

Problem 4. Compute the subgroup of $\operatorname{Jac}\left(W_{44}\right)$ generated by divisors supported at the cusps and points of order two.

Algebraic geometers and number theorists have been interested in exhibiting explicit examples of algebraic curves whose Jacobians have endomorphisms. A parallel goal is to exhibit Riemann surfaces whose Jacobians have endomorphisms as polygons in the plane glued together by translations as we did for the complex multiplication points on $\mathcal{M}_{2}\left(D_{8}\right)$ and $\mathcal{M}_{2}\left(D_{12}\right)$.

Problem 5. Exhibit surfaces whose Jacobians have complex multiplication as polygons in $\mathbb{C}$ glued together by translation.

Notes and references. For a survey of results related to the Teichmüller geodesic flow, Teichmüller curves and relations to billiards see [KMS, MT, KZ, Zo]. Background about abelian varieties, Hilbert modular surfaces and Shimura varieties can be found in [vdG], [Sh2], [BL], and [Sh1]. The orbifold points on $X_{D}$ are studied in [Pr] and the family $\mathcal{M}_{2}\left(D_{8}\right)$ was studied in [Si]. 


\section{Chapter 2}

\section{Background}

\subsection{Abelian varieties}

In this section we will collect background and definitions about abelian varieties, endomorphisms of abelian varieties, Shimura varieties, Hilbert modular surfaces and modular curves. For more background on abelian varieties, endomorphisms and Shimura varieties see $[\mathrm{BL}]$, for more background on Hilbert modular surfaces see [vdG] and for more background on modular curves see [DS].

Abelian varieties. A $g$-dimensional complex torus $B=V / \Lambda$ is the quotient of a $g$-dimensional $\mathbb{C}$-vector space $V$ by a cocompact lattice $\Lambda$. Any connected, compact complex Lie group is a complex torus. The vector space $V$ is naturally isomorphic to the dual to the space of holomorphic one forms on $B$ and the lattice $\Lambda$ is naturally isomorphic to the first integral homology:

$$
B \cong \Omega(B)^{*} / H_{1}(B, \mathbb{Z})
$$

A polarization on $B$ is the first Chern class of an ample line bundle on $A$, or equivalently, an integral symplectic form $E_{B}(\cdot, \cdot)$ on $H_{1}(B, \mathbb{Z})$ with the property that $E_{B}(i \cdot, \cdot)+i E_{B}(\cdot, \cdot)$ is a positive definite Hermitian form on $\Omega(B)^{*} \cong H_{1}(B, \mathbb{Z}) \otimes \mathbb{R}$. A complex torus is an abelian variety if it admits a polarization. A polarization on 
$B$ is principal if the symplectic form is unimodular on $H_{1}(B, \mathbb{Z})$. We will denote by $A_{g}$ the moduli space of principally polarized abelian varieties of dimension $g$.

Homomorphisms and endomorphisms of abelian varieties. A homomorphism of abelian varieties $f: B_{1} \rightarrow B_{2}$ is a holomorphic map which is also a homomorphism. Any holomorphic map between abelian varieties is the composition of a homomorphism with a translation. A homomorphism of abelian varieties $f$ is determined by its lift to the universal cover, which is a $\mathbb{C}$-linear map $\tilde{f}: \Omega\left(B_{1}\right)^{*} \rightarrow \Omega\left(B_{2}\right)^{*}$ with $\widetilde{f}\left(H_{1}(A, \mathbb{Z})\right) \subset H_{1}(B, \mathbb{Z})$. The map $f$ is an isogeny if it has finite kernel and cokernel. Principal polarizations on $B_{1}$ and $B_{2}$ determine a dual map $f^{*}: B_{2} \rightarrow B_{1}$ characterized by:

$$
E_{B_{2}}(\tilde{f} x, y)=E_{B_{1}}\left(x, \widetilde{f}^{*} y\right)
$$

whenever $x \in H_{1}\left(B_{1}, \mathbb{Z}\right)$ and $y \in H_{1}\left(B_{2}, \mathbb{Z}\right)$.

Now fix a principally polarized abelian variety $B$. The endomorphisms of $B$ are the holomorphic self-homomorphisms and form a $\mathbb{Z}$-algebra $\operatorname{End}(B)$ called the endomorphism ring of $B$. An endomorphism $f$ of $B$ is an automorphism if it preserves the polarization, i.e. $f^{*}=f^{-1}$. The rational endomorphism ring of $B$ is the $\mathbb{Q}$-algebra $\operatorname{End}(B) \otimes \mathbb{Q}$. The ring $\operatorname{End}(B)$ is a subring with unit of $\operatorname{End}(B) \otimes \mathbb{Q}$ which is also a lattice, i.e. an order in $\operatorname{End}(B) \otimes \mathbb{Q}$. The ring $\operatorname{End}(B)$ has an analytic representation $\Phi_{a}$ on $\Omega(B)^{*}$, a rational representation $\Phi_{r}$ on $H_{1}(B, \mathbb{Q})$ and a representation $\Phi_{\mathbb{Q}}$ on the $\mathbb{Q}$-vector space $\operatorname{End}(B) \otimes \mathbb{Q}$. The anti-involution $f \mapsto f^{*}$ of $\operatorname{End}(B)$ is called the Rosati involution. The Rosati involution is positive in the sense that $(f, g) \mapsto \operatorname{Tr}\left(\Phi_{\mathbb{Q}}\left(f g^{*}\right)\right)$ is a positive definite bilinear form.

Every abelian variety has a self-dual action of $\mathbb{Z}$ and a typical abelian variety has $\operatorname{End}(B)=\mathbb{Z}$. The subgroup $B[n]$ of $B$ is the kernel of the multiplication by $n$ map and $B[n]^{*}$ is set of points of order $n$. The endomorphism ring of the product of elliptic 
curves $E \times F$ is given by:

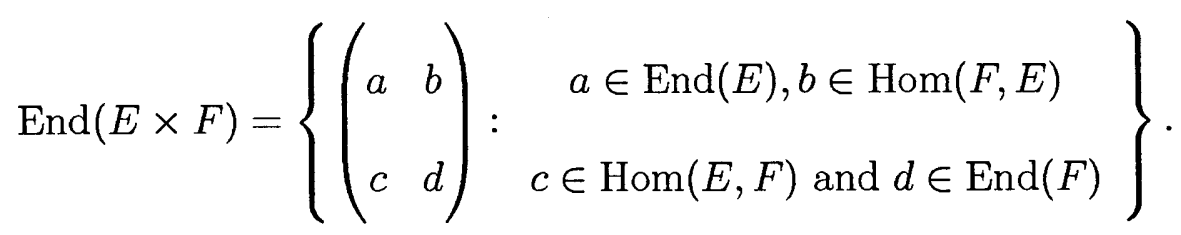

The Rosati involution induced by the product polarization is $\left(\begin{array}{ll}a & b \\ c & d\end{array}\right)^{*}=\left(\begin{array}{ll}a^{*} & c^{*} \\ b^{*} & d^{*}\end{array}\right)$.

Families of abelian varieties with endomorphisms. Let $\mathcal{O}$ be a $\mathbb{Z}$-algebra with positive anti-involution $*$. An $\mathcal{O}$-action on an abelian variety $B \in A_{g}$ is an injective homomorphism:

$$
\iota: \mathcal{O} \rightarrow \operatorname{End}(B) \text { with } \iota(x)^{*}=\iota\left(x^{*}\right)
$$

The $\mathcal{O}$-action $\iota$ is proper if it does not extend to a larger ring in $\mathcal{O} \otimes \mathbb{Q}$. The family:

$$
A_{\boldsymbol{g}}(\mathcal{O})=\{(B, \iota): \iota \text { is a proper } \mathcal{O} \text {-action on } B\} / \sim
$$

parametrizes principally polarized abelian varieties with an $\mathcal{O}$-action. Two pairs $\left(B_{1}, \iota_{1}\right)$ and $\left(B_{2}, \iota_{2}\right)$ are equivalent, i.e. $\left(B_{1}, \iota_{1}\right) \sim\left(B_{2}, \iota_{2}\right)$, if there is an isomorphism of polarized abelian varieties $B_{1} \rightarrow B_{2}$ intertwining $\iota_{i}$.

The components of $A_{g}(\mathcal{O})$ can be distinguished by the rational representation of $\mathcal{O}$. A unimodular $\mathcal{O}$-module is an $\mathcal{O}$-module $M$ with a unimodular symplectic form $E_{M}(\cdot, \cdot)$ which is positive, i.e. the trace of $\left(x x^{*}\right) \in \operatorname{End}(M)$ is positive for reach $x \in \mathcal{O}$, and which is compatible with $*$, i.e. $E_{M}\left(x m_{1}, m_{2}\right)=E_{M}\left(m_{1}, x^{*} m_{2}\right)$ for every $x \in \mathcal{O}$ and $m_{i} \in M$. A unimodular $\mathcal{O}$-module $M$ is an ideal if it is a submodule of $\mathcal{O} \otimes \mathbb{Q}$ and $M$ is proper if the action of $\mathcal{O}$ does not extend to a larger ring in $\mathcal{O} \otimes \mathbb{Q}$. A proper, unimodular $\mathcal{O}$-module $M$ with $\mathbb{Z}$-rank $2 g$ determines a component of $A_{g}(\mathcal{O})$ :

$$
A_{g}(M)=\left\{(B, \iota, \phi): \quad \begin{array}{cc}
(B, \iota) \in A_{g}(\mathcal{O}) \text { and } \phi: M \rightarrow H_{1}(B, \mathbb{Z}) \\
& \text { is a symplectic } \mathcal{O} \text {-module isomorphism }
\end{array}\right\} / \sim
$$

The triple $\left(B_{1}, \iota_{1}, \phi_{1}\right)$ is equivalent to $\left(B_{2}, \iota_{2}, \phi_{2}\right)$ if there is an isomorphism $B_{1} \rightarrow B_{2}$ 
intertwining $\iota_{i}$ and $\phi_{i}$.

Shimura varieties. A Shimura variety is the quotient of a Hermitian symmetric domain by an arithmetic group. The family $A_{g}(M)$ is a prototypical example of a Shimura variety. Complex structures on $M \otimes_{\mathbb{Z}} \mathbb{R}$ compatible with $E_{M}$ form a Hermitian symmetric domain $\mathcal{H}(M)$. A point $\tau \in \mathcal{H}(M)$ turns the real torus $M \otimes_{\mathbb{Z}}$ $\mathbb{R} / M$ into an abelian variety $B_{\tau}$ with $\mathcal{O}$-action $\iota_{\tau}$. Two complex structures in $\mathcal{H}(M)$ give the same point in $A_{g}(M)$ if they differ by an element of the arithmetic group $\mathrm{SL}(M)$ of symplectic $\mathcal{O}$-module automorphisms $M$.

Let $G$ be the subgroup of $\operatorname{SL}(M)$ which fixes every point in $\mathcal{H}(M)$. The group $G$ acts by automorphisms commuting with $\mathcal{O}$ on each abelian variety in $A_{g}(M)$ and the group $\operatorname{PSL}(M)=\mathrm{SL}(M) / G$ acts faithfully on $\mathcal{H}(M)$. The space $A_{g}(M)$ is presented as a complex orbifold and Shimura variety by:

$$
A_{g}(M) \cong \mathcal{H}(M) / \operatorname{PSL}(M)
$$

Real and complex multiplication. Let $L$ be a commutative $\mathbb{Q}$-algebra with positive anti-involution $*$ and let $L^{0}$ denote the set of self-adjoint elements of $L$. Since * is positive, $L^{0}$ is a direct sum of totally real fields and either $L=L^{0}$ or $L$ is a degree two totally imaginary extension of $L^{0}$ (see [Sh1], Prop. 1).

When $L=L^{0}$ and $\mathcal{O}$ is an order in $L$, we will say that a $g=[L: \mathbb{Q}]$ dimensional abelian variety $B \in A_{g}$ has real multiplication by $\mathcal{O}$ if $B$ admits a proper $\mathcal{O}$-action. For a proper unimodular module $M$ over $\mathcal{O}$, the space $\mathcal{H}(M)$ is isomorphic to $(\mathbb{H})^{g}$ and the group $\mathrm{SL}(M)$ is commensurable to $\mathrm{SL}_{2}(\mathcal{O})$.

When $\left[L: L^{0}\right]=2,[L: \mathbb{Q}]=2 g$ and $\mathcal{O}$ is an order in $L$, we will say $B \in A_{g}$ has complex multiplication by the order $\mathcal{O}$ if $B$ admits a proper $\mathcal{O}$-action. For a proper unimodular module $M$ over $\mathcal{O}$, the space $\mathcal{H}(M)$ is a point.

Real quadratic orders. Each integer $D \equiv 0$ or 1 mod 4 determines a quadratic ring:

$$
\mathcal{O}_{D}=\frac{\mathbb{Z}[t]}{\left(t^{2}-D t+D(D-1) / 4\right)}
$$


Every quadratic ring is isomorphic to $\mathcal{O}_{D}$ for some $D$. The integer $D$ is called the discriminant of $\mathcal{O}_{D}$ and $\mathcal{O}_{D}$ is totally real whenever $D>0$. Let $K_{D}=\mathcal{O}_{D} \otimes \mathbb{Q}$, let $\mathcal{O}_{K_{D}}$ be the maximal order in $K_{D}$ and let $\sqrt{D}$ be the square root of $D$ equal to $2 t-D$. The conductor $f$ of $\mathcal{O}_{D}$ is the index of $\mathcal{O}_{D}$ in $\mathcal{O}_{K_{D}}$. The inverse different $\mathcal{O}_{D}^{\vee}=\frac{1}{\sqrt{D}} \mathcal{O}_{D}$ is the fractional ideal dual to $\mathcal{O}_{D}$ under the trace pairing. When $D>0$, let $\sigma_{+}$and $\sigma_{-}$denote the two homomorphisms $K_{D} \rightarrow \mathbb{R}$ satisfying $\sigma_{+}(\sqrt{D})>0>\sigma_{-}(\sqrt{D})$. We will also write $\sigma_{+}$and $\sigma_{-}$for the induced homomorphisms $M_{2}\left(K_{D}\right) \rightarrow M_{2}(\mathbb{R})$ and $\mathrm{SL}_{2}\left(K_{D}\right) \rightarrow \mathrm{SL}_{2}(\mathbb{R})$.

Our analysis works equally well when $D$ is a square, even though the $\operatorname{ring} \mathcal{O}_{D}$ is no longer a domain and the homomorphisms $\sigma_{ \pm}$are no longer injective. When $D=f^{2}$, we have $K_{D}=\mathbb{Q} \times \mathbb{Q}, \mathcal{O}_{K_{D}}=\mathbb{Z} \times \mathbb{Z}$ and $\mathcal{O}_{D}$ is the subring of $\mathbb{Z} \times \mathbb{Z}$ consisting of pairs $(a, b)$ with $a \equiv b \bmod f$.

Hilbert modular surfaces. The $\mathcal{O}_{D}$-module $\mathcal{O}_{D} \oplus \mathcal{O}_{D}^{\vee}$ has a unimodular symplectic form:

$$
\left\langle\left(x_{1}, y_{1}\right),\left(x_{2}, y_{2}\right)\right\rangle=\operatorname{Tr}_{\mathbb{Q}}^{K}\left(x_{1} y_{2}-x_{2} y_{1}\right) .
$$

Up to isomorphism of symplectic $\mathcal{O}_{D}$-modules, $\mathcal{O}_{D} \oplus \mathcal{O}_{D}^{\vee}$ is the unique unimodular and proper $\mathcal{O}_{D}$-module isomorphic to $\mathbb{Z}^{4}$ as a $\mathbb{Z}$-module. The Hermitian symmetric domain $\mathcal{H}\left(\mathcal{O}_{D} \oplus \mathcal{O}_{D}^{\vee}\right)$ is isomorphic to $\mathbb{H} \times \mathbb{H}$ with a point $\tau=\left(\tau_{1}, \tau_{2}\right)$ corresponding to the abelian variety:

$$
B_{\tau}=\mathbb{C}^{2} / \phi_{\tau}\left(\mathcal{O}_{D} \oplus \mathcal{O}_{D}^{\vee}\right)
$$

where $\phi_{\tau}(x, y)=\left(\sigma_{+}(x)+\tau_{1} \sigma_{+}(y), \sigma_{-}(x)+\tau_{2} \sigma_{-}(y)\right)$. The diagonal action $x \mapsto$ $\left(\begin{array}{cc}\sigma_{+}(x) & 0 \\ 0 & \sigma_{-}(x)\end{array}\right)$ of $\mathcal{O}_{D}$ on $\mathbb{C}^{2}$ covers a proper $\mathcal{O}_{D}$-action $\iota_{\tau}$ on $B_{\tau}$, giving a point $\left(B_{\tau}, \iota_{\tau}\right) \in$ $A_{2}\left(\mathcal{O}_{D} \oplus \mathcal{O}_{D}^{\vee}\right)$. Two points in $\mathbb{H} \times \mathbb{H}$ determine the same point in $A_{g}\left(\mathcal{O}_{D} \oplus \mathcal{O}_{D}^{\vee}\right)$ if and only if they differ by an element of the group:

$$
\operatorname{SL}\left(\mathcal{O}_{D} \oplus \mathcal{O}_{D}^{\vee}\right)=\left\{\left(\begin{array}{cc}
a & b \\
c & d
\end{array}\right): \begin{array}{c}
a d-b c=1, a, d \in \mathcal{O}_{D}, \\
b \in \sqrt{D} \mathcal{O}_{D} \text { and } c \in \mathcal{O}_{D}^{\vee}
\end{array}\right\}
$$


The matrix $A \in \operatorname{SL}\left(\mathcal{O}_{D} \oplus \mathcal{O}_{D}^{\vee}\right)$ acts on $\mathbb{H} \times \mathbb{H}$ by $A \cdot\left(\tau_{1}, \tau_{2}\right)=\left(\sigma_{+}(A) \tau_{1}, \sigma_{-}(A) \tau_{2}\right)$ where the matrices $\sigma_{ \pm}(A) \in \mathrm{SL}_{2}(\mathbb{R})$ act on $\mathbb{H}$ by linear fractional transformation. The group $G$ fixing every point in $\mathbb{H} \times \mathbb{H}$ is generated by the elements of order two and $G$ has order two unless $D=1$ or 4 , in which case $G$ has order four. The Hilbert modular surface:

$$
X_{D}=(\mathbb{H} \times \mathbb{H}) / \operatorname{PSL}\left(\mathcal{O}_{D} \oplus \mathcal{O}_{D}^{\vee}\right)
$$

is in natural bijection with the points in the Shimura variety $A_{2}\left(\mathcal{O}_{D} \oplus \mathcal{O}_{D}^{\vee}\right)$ and presents $A_{2}\left(\mathcal{O}_{D} \oplus \mathcal{O}_{D}^{\vee}\right)$ as a complex orbifold.

Quaternion algebras. Now fix two positive discriminants $D$ and $E$. Let $\mathcal{O}$ be an order in the quaternion algebra:

$$
\left(\frac{D, E}{\mathbb{Q}}\right)=\mathbb{Q}\langle a, b\rangle /\left(a^{2}=D, b^{2}=E, a b=-b a\right),
$$

which is invariant under the anti-involution generated by $a^{*}=a, b^{*}=b$ and $(a b)^{*}=b a$ and has $\mathcal{O} \cap \mathbb{Q}(a)=\mathcal{O}_{D}$ and $\mathcal{O} \cap \mathbb{Q}(b)=\mathcal{O}_{E}$. Let $M$ be a proper, unimodular $\mathcal{O}$-module. The family $A_{2}(M)$ parametrizes a family of abelian varieties with real multiplication by $\mathcal{O}_{D}$ and $\mathcal{O}_{E}$ and the projection $A_{2}(M) \rightarrow A_{2}$ covers a component of the intersection of the image of $X_{D}$ with the image of $X_{E}$. The Hermitian symmetric domain $\mathcal{H}(M)$ is isomorphic to $\mathbb{H}$ and the arithmetic group $\mathrm{SL}(M)$ is isomorphic to the group of units in $\mathcal{O}$.

Square discriminants. When $D$ is a square, the Hilbert modular surface $X_{D}$ parametrizes abelian varieties which are isogenous to a product of elliptic curves. The Hilbert modular surface $X_{1}$ is isomorphic to $\mathcal{M}_{1} \times \mathcal{M}_{1}$ and parametrizes abelian varieties which are isomorphic to products of elliptic curves.

Elliptic transformations and orbifold points. An element $A \neq 1$ in $\operatorname{PSL}(M)$ is elliptic if it fixes a point in $\mathcal{H}(M)$. For $\tau \in \mathcal{H}(M)$, let $\operatorname{Stab}(\tau)$ denote the stablizer of $\tau$ in $\operatorname{SL}(M)$. The group $\operatorname{Stab}(\tau)$ acts by automorphisms commuting with $\iota_{\tau}(\mathcal{O})$ on the associated abelian variety $B_{\tau}$. When $\operatorname{Stab}(\tau)$ is strictly larger than $G$, the point 
$\left(B_{\tau}, \iota_{\tau}\right) \in A_{g}(M)$ is called an orbifold point and the cardinality of $\operatorname{Stab}(\tau) / G$ is called the orbifold order of $\left(B_{\tau}, \iota_{\tau}\right)$.

An elliptic transformation in $A \in \operatorname{PSL}\left(\mathcal{O}_{D} \oplus \mathcal{O}_{D}^{\vee}\right)$ fixes either a unique point in $\mathbb{H} \times \mathbb{H}$ or every point on a complex geodesic of the form $\tau \times \mathbb{H}$ or $\mathbb{H} \times \tau$. It is easy to check that, when $D \geq 4$, the elliptic elements of $\operatorname{PSL}\left(\mathcal{O}_{D} \oplus \mathcal{O}_{D}^{\vee}\right)$ have unique fixed points. For such discriminants, the following are equivalent:

- A has finite order,

- $\left|\operatorname{Tr}\left(\sigma_{+}(A)\right)\right|<2$ and $\left|\operatorname{Tr}\left(\sigma_{-}(A)\right)\right|<2$,

- $A$ fixes a point in $\mathbb{H} \times \mathbb{H}$, and

- $A$ fixes a unique point in $\mathbb{H} \times \mathbb{H}$.

There is a natural bijection between the elements of order $n>2$ in $\operatorname{SL}\left(\mathcal{O}_{D} \oplus \mathcal{O}_{D}^{\vee}\right)$ up to conjugacy and abelian varieties with complex multiplication by orders $\mathcal{O}$ containing $\mathcal{O}_{D}\left[\zeta_{n}\right]$ with $\mathcal{O} \cap\left(\mathcal{O}_{D} \otimes \mathbb{Q}\right)=\mathcal{O}_{D}$ and $\zeta_{n}$ a primitive $n$th root of unity.

Modular curves. The modular curve $\mathcal{M}_{1} \cong A_{1}$ is the moduli space of elliptic curves and is isomorphic to the hyperbolic Riemann surface $\mathbb{H} / \mathrm{SL}_{2}(\mathbb{Z})$. A number $\tau \in \mathbb{H}$ corresponds to the elliptic curve $E_{\tau}=\mathbb{C} / \mathbb{Z} \oplus \tau \mathbb{Z}$. Let $\Gamma(n)$ be the kernel of the natural homomorphism $\mathrm{SL}_{2}(\mathbb{Z}) \rightarrow \mathrm{SL}_{2}(\mathbb{Z} / n \mathbb{Z})$, let $\Gamma_{1}(n)=\left\langle\Gamma(n),\left(\begin{array}{ll}1 & 1 \\ 0 & 1\end{array}\right)\right\rangle$ and let $\Gamma_{0}(n)=\left\{\left(\begin{array}{cc}a & b \\ c & d\end{array}\right): c \equiv 0 \bmod n\right\}$. The quotients:

$$
Y(n)=\mathbb{H} / \Gamma(n), Y_{1}(n)=\mathbb{H} / \Gamma_{1}(n) \text { and } Y_{0}(n)=\mathbb{H} / \Gamma_{0}(n),
$$

parametrize elliptic curves distinguished points of order $n$. Note that $\Gamma_{0}(2)=\Gamma_{1}(2)$.

We will be most concerned with the curve $Y_{0}(n)$ which parametrizes cyclic degree$n$ isogenies $i: E \rightarrow F$ between elliptic curves up to isomorphism (see Figure 4-1). The point $\tau \in \mathbb{H}$ corresponds to the isogeny $i_{\tau}$ on $E_{\tau}$ whose kernel is generated by the image $T_{\tau}$ of $1 / n$ in $\mathbb{C} / \mathbb{Z} \oplus \tau \mathbb{Z}$. The modular curve $Y_{0}(n)$ has an Atkin-Lehner involution sending the isogeny $i_{\tau}$ to its dual $i_{\tau}^{*}=i_{-1 / n \tau}$. 
Class numbers of imaginary quadratic fields. Fix a negative discriminant $D<$ 0 . The following sets are in natural bijection:

- Elliptic curves with complex multiplication by $\mathcal{O}_{D}$ up to isomorphism,

- Proper $\mathcal{O}_{D^{-}}$-ideal classes, and

- Triples of integers $(a, b, c)$ with $D=b^{2}-4 a c, \operatorname{gcd}(a, b, c)=1,|b| \leq a \leq c$, and if $|b|=a$ or $a=c$ then $b \geq 0$.

The triple $(a, b, c)$ corresponds to the ideal class of $I=a \mathbb{Z} \oplus \frac{-b+\sqrt{D}}{2} \mathbb{Z}$, which in turn corresponds to the elliptic curve $\mathbb{C} / I$. The conditions on $(a, b, c)$ ensure that $I$ is a proper $\mathcal{O}_{D}$-ideal and the number $\tau=(-b+\sqrt{D}) /(2 a)$ is in the standard fundamental domain for the action of the group $\mathrm{SL}_{2}(\mathbb{Z})$ on $\mathbb{H}$.

The class number $h(-D)$ is the cardinality the set of $\mathcal{O}_{D}$-ideal classes. Using $|D|=4 a c-b^{2} \geq 4 a^{2}-a^{2}$, we have that $|b|, a<\sqrt{D} / 3$ and (cf. [Coh], pg. 232):

$$
h(-D)<2 D / 3
$$

\subsection{Riemann surfaces, Jacobians and Automorphisms}

In this section we will collect background and definitions about the moduli space of curves, Jacobians, the period map and the Teichmüller geodesic flow. References for this section include [BL] (especially §11) for Jacobians and the period map and [MT] and $[\mathrm{Zo}]$ for the Teichmüller geodesic flow.

Jacobians of Riemann surfaces. Let $\mathcal{M}_{g}$ denote the moduli space of genus $g$ Riemann surfaces. The space $\mathcal{M}_{g}$ is both an algebraic variety and a complex orbifold. The space $\mathcal{M}_{g}$ has a Deligne-Mumford compactification $\overline{\mathcal{M}}_{g}$ by stable algebraic curves.

For $X \in \mathcal{M}_{g}$, let $\Omega(X)$ denote space of holomorphic one forms on $X$. The Jacobian of $X$ is the $g$-dimensional principally polarized abelian variety:

$$
\operatorname{Jac}(X)=\Omega(X)^{*} / H_{1}(X, \mathbb{Z})
$$


A holomorphic map $f: X \rightarrow Y$ between Riemann surfaces induces a holomorphic homomorphism $\operatorname{Jac}(X) \rightarrow \operatorname{Jac}(Y)$. The automorphisms of $X$ give automorphisms of the polarized abelian variety $\operatorname{Jac}(X)$ and when $g=2$, the automorphism groups coincide $\operatorname{Aut}(X)=\operatorname{Aut}(\operatorname{Jac}(X))$.

A point $P \in X$ determines a map $\theta_{P}: X \rightarrow \operatorname{Jac}(X)$ and any holomorphic map from $X$ to an abelian variety $B$ factors through $\theta_{P}$. The $\operatorname{Jacobian} \operatorname{Jac}(X)$ is isomorphic to the identity component $\operatorname{Pic}^{0}(X)$ of the Picard variety. The abelian variety $\operatorname{Pic}^{0}(X)$ parametrizes degree zero line bundles on $X$.

The period map. The period map $X \mapsto \operatorname{Jac}(X)$ embeds $\mathcal{M}_{g}$ in $A_{g}$ and extends to the moduli space $\widetilde{\mathcal{M}}_{g} \subset \overline{\mathcal{M}}_{g}$ of stable algebraic curves with compact Jacobians. The boundary of $\mathcal{M}_{g}$ in $\widetilde{\mathcal{M}}_{g}$ consists of polarized products of Jacobians of smooth curves of lower genus.

When $g \leq 2$, the period map is dominant and extends to an isomorphism of complex orbifolds on $\widetilde{\mathcal{M}}_{g}$. The space $\widetilde{\mathcal{M}}_{2}$ is the partial compactification of $\mathcal{M}_{2}$ by the moduli space of pairs of elliptic curves $E \vee F$ joined at a single simple node:

$$
\widetilde{\mathcal{M}}_{2}=\mathcal{M}_{2} \cup \operatorname{Sym}^{2}\left(\mathcal{M}_{1}\right)
$$

The Jacobian of $E \vee F$ is the product $E \times F$ with the product polarization. The complement of $\mathcal{M}_{2}$ in $A_{2}$ is the image of the Hilbert modular surface $X_{1}$ :

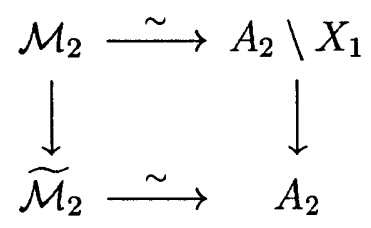

Surfaces with automorphisms. For a finite group $G$, the family of genus $g$ surfaces with a faithful action of $G$ is parametrized by the space:

$$
\widetilde{\mathcal{M}}_{g}(G)=\left\{(X, \rho): \begin{array}{l}
X \in \widetilde{\mathcal{M}}_{g}, \\
\text { and } \rho: G \rightarrow \operatorname{Aut}(X) \\
\text { is injective. }
\end{array}\right\} / \sim .
$$


Two pairs $\left(X_{1}, \rho_{1}\right)$ and $\left(X_{2}, \rho_{2}\right)$ are equivalent, i.e. $\left(X_{1}, \rho_{1}\right) \sim\left(X_{2}, \rho_{2}\right)$, if there is an isomorphism $X_{1} \rightarrow X_{2}$ intertwining the $\rho_{i}$. The set $\widetilde{\mathcal{M}}_{g}(G)$ has a natural topology and a unique holomorphic structure making the map $\widetilde{\mathcal{M}}_{g}(G) \rightarrow \mathcal{M}_{g}$ holomorphic.

We will denote by $\mathcal{M}_{g}(G)$ the subspace of $\widetilde{\mathcal{M}}_{g}(G)$ parametrizing the family of smooth surfaces with an action of $G$. The action of $G$ on $\operatorname{Jac}(X)$ extends to a (not necessarily faithful) action of the group ring $\mathbb{Z}[G]$. The Rosati-involution is generated by $g^{*}=g^{-1}$ for $g \in G$.

An injective group homomorphism $G_{1} \rightarrow G_{2}$ gives a holomorphic map $\widetilde{\mathcal{M}}_{2}\left(G_{2}\right) \rightarrow$ $\widetilde{\mathcal{M}}_{2}\left(G_{1}\right)$. The automorphism group of $G$ acts on $\widetilde{\mathcal{M}}_{2}(G)$ and the inner automorphisms act trivially. This gives an action of the outer automorphism group $\operatorname{Out}(G)=$ $\operatorname{Aut}(G) / \operatorname{Inn}(G)$ on $\widetilde{\mathcal{M}}_{2}(G)$. Note that $\operatorname{Out}\left(D_{8}\right)$ is isomorphic to $\mathbb{Z} / 2 \mathbb{Z}$ and the nontrivial outer automorphism $\sigma$ has $\sigma(r)=J r$ and $\sigma(J)=J$.

Correspondences. Suppose $f: Y \rightarrow X_{1}$ and $g: Y \rightarrow X_{2}$ are holomorphic maps between Riemann surfaces and $f_{*}: \operatorname{Jac}(Y) \rightarrow \operatorname{Jac}\left(X_{1}\right)$ and $g_{*}: \operatorname{Jac}(Y) \rightarrow \operatorname{Jac}\left(X_{2}\right)$ are the maps induced on Jacobians. The map $Y \rightarrow X_{1} \times X_{2}$ is called a correspondence between $X_{1}$ and $X_{2}$ and induces a holomorphic homomorphism $g_{*}\left(f_{*}\right)^{*}: \operatorname{Jac}\left(X_{1}\right) \rightarrow$ $\operatorname{Jac}\left(X_{2}\right)$.

Under an identification of $\operatorname{Jac}\left(X_{i}\right)$ with $\operatorname{Pic}^{0}\left(X_{i}\right)$, the holomorphic homomorphism $g_{*}\left(f_{*}\right)^{*}$ is obtained by taking a degree zero divisor on $X_{1}$, pulling it back to $Y$ and pushing it forward to $X_{2}$ :

$$
\left(g_{*}\right)\left(f_{*}\right)^{*}: \sum_{i}\left(P_{i}-Q_{i}\right) \mapsto \sum_{i} g\left(f^{-1}\left(P_{i}\right)\right)-g\left(f^{-1}\left(Q_{i}\right)\right),
$$

for any points $P_{i}$ and $Q_{i}$ are points in $X_{1}$.

The hyperelliptic involution and Weierstrass points. Every genus two surface with compact Jacobian $X \in \widetilde{\mathcal{M}}_{2}$ has a hyperelliptic involution $\eta$ acting by $\omega \mapsto-\omega$ on $\Omega(X)$.

When $X$ is smooth, the quotient $X / \eta$ is a sphere naturally isomorphic to $\mathbb{P} \Omega(X)^{*}$. The surface $X$ has six distinguished Weierstrass points fixed by $\eta$ and we will denote 
the set of Weierstrass points by $X^{W}=\operatorname{Fix}(\eta)$. For each $P \in X$, there is a holomorphic one form in $\Omega(X)$ with zeros at $P$ and $\eta(P)$ and the Weierstrass points are the zeros of holomorphic one-forms with double zero.

When $X=E_{1} \vee E_{2}$ is singular, $\eta$ restricts to the elliptic involution on each component of $X$ fixing the node. Taking the node to be the base point for $E_{1}$ and $E_{2}$, the set of Weierstrass points $X^{W}=E_{1}[2]^{*} \sqcup E_{2}[2]^{*}$ consists of the points of order two. The node is not a Weierstrass point even though it is fixed by $\eta$.

For any $X \in \widetilde{\mathcal{M}}_{2}$, the hyperelliptic involution $\eta$ is in the center of the automorphism group $\operatorname{Aut}(X)$ and the group $\operatorname{Aut}(X)$ acts naturally on both $X^{W}$ and $X / \eta$. The group $\operatorname{Sym}\left(X^{W}\right)$ is isomorphic to the symmetric group on six letters $S_{6}$ and the conjugacy classes in $\operatorname{Sym}\left(X^{W}\right)$ are naturally labeled by partitions of six:

$$
\left[n_{1}, \ldots, n_{k}\right] \text { with } \sum_{i} n_{i}=6
$$

Involutions of genus two surfaces. Fix an order two automorphism $\phi$ on a genus two surface $X \in \widetilde{\mathcal{M}}_{2}$. There are three possibilities for $\phi$, distinguished by the action of $\mathbb{Z}[\phi] \cong \mathcal{O}_{4}$ on $\operatorname{Jac}(X)$ :

- $\mathbb{Z}[\phi]$ is not faithful. The map $\phi$ acts by -1 on $\operatorname{Jac}(X)$ and $\phi$ is the hyperelliptic involution.

- $\mathbb{Z}[\phi]$ is faithful but not proper. The action of $\mathbb{Z}[\phi]$ on $\operatorname{Jac}(X)$ extends to real multiplication by the maximal order $\mathcal{O}_{1}=\mathbb{Z}\left[\frac{1+\phi}{2}\right]$. The surface $X$ is nodal and $\phi$ restricts to the identity on one component of $X$ and the elliptic involution on the other. The eigenspaces for $\phi$ in $\Omega(X)$ are one dimensional and $\phi$ fixes the components of $X$.

- $\mathbb{Z}[\phi]$ is proper. The quotient $X / \phi$ has genus one. This is true for nodal $X$ since $\phi$ interchanges the components of $X$ because it does not commute with $\mathcal{O}_{1}$. For smooth $X, \phi$ is an involution different from the hyperelliptic involution, so the quotient must have genus one. The eigenspaces for $\phi$ on $\Omega(X)$ are onedimensional and $\operatorname{Jac}(X)$ has real multiplication by $\mathcal{O}_{4}=\mathbb{Z}[\phi]$. 
Fix a surface $X$ with involution $\phi$ such that the induced action of $\mathbb{Z}[\phi]$ on $\operatorname{Jac}(X)$ is proper. Since $\phi$ commutes with the hyperelliptic involution $\eta, \eta$ induces an elliptic involution $\eta_{E}$ on the quotient $E=X / \phi$. It is easy to check that $\operatorname{Fix}(\eta \phi)$ forms a single $\phi$-orbit and that $\eta_{E}$ fixes its image. Setting this point to be the base point, i.e. $E[1]=$ $\operatorname{Fix}(\eta \phi) / \phi$, identifies $E$ with its Jacobian via $\theta_{E[1]}: E \rightarrow \operatorname{Jac}(E)$. The remaining points fixed by $\eta_{E}$ are covered by the Weierstrass points, giving an identification:

$$
E[2]^{*}=X^{W} / \phi
$$

Similarly, the quotient $F=X / \eta \phi$ has genus one, a natural elliptic involution $\eta_{F}$, base point $F[1]=\operatorname{Fix}(\phi) / \eta \phi$ and identification $F[2]^{*}=X^{W} / \eta \phi$.

Abelian and quadratic differentials. For a Riemann surface $X \in \mathcal{M}_{g}$, the space of holomorphic quadratic differentials $Q(X)$ consists of sections of the square of the cotangent bundle on $X$. The space of holomorphic one-forms on $X$ admits a quadratic map $\Omega(X) \rightarrow Q(X)$ and when $g \leq 3$, the space $\operatorname{Sym}^{2}(\Omega(X))$ is naturally isomorphic to $Q(X)$. The bundle of abelian differentials $\Omega \mathcal{M}_{g} \rightarrow \mathcal{M}_{g}$ parametrizes Riemann surfaces with a non-zero holomorphic one-form. The bundle of quadratic differentials $Q \mathcal{M}_{g} \rightarrow \mathcal{M}_{g}$ parametrizes Riemann surfaces with a non-zero quadratic differential and is isomorphic to the complement of the zero section in the cotangent bundle $T^{*} \mathcal{M}_{g}$

A typical way to give a point in $(X, q) \in Q \mathcal{M}_{g}$ is to glue polygons $P_{\boldsymbol{i}}$ in $\mathbb{C}$ together along their sides by affine maps of the form $z \mapsto \pm z+c$ as in Figures 1-2 and 1-3. The quadratic differential $d z^{2} \in Q(\mathbb{C})$ is invariant under such maps and gives a quadratic differential on the quotient:

$$
(X, q)=\bigcup_{i}\left(P_{i}, d z^{2}\right) / \sim
$$

A quadratic differential $(X, q)$ is the square of a one-form, i.e. $q=\omega^{2}$, if $(X, q)$ can be written as a union of polygons glued together by affine maps of the form $z \mapsto z+c$. 
Complex geodesics. The real geodesic flow on $\mathcal{M}_{g}$ gives $\mathbb{R}$-actions on $Q \mathcal{M}_{g}$ and $\Omega \mathcal{M}_{g}$ which extend to $\mathrm{SL}_{2}(\mathbb{R})$-actions. For the quadratic differential $(X, q)=$ $\bigcup_{i}\left(P_{i}, d z^{2}\right) / \sim$ obtained from polygons $P_{i} \subset \mathbb{C}$, the matrix $A \in \mathrm{SL}_{2}(\mathbb{R})$ acts on $\mathbb{C}$ by real-linear transformations giving a new surface with quadratic differential $A \cdot(X, q)=\bigcup_{i}\left(A\left(P_{i}\right), d z^{2}\right) / \sim$.

The subgroup $\mathrm{SO}_{2}(\mathbb{R})$ acts on $(X, q)$ by leaving $X$ fixed and rotating $q$. The $\mathrm{SL}_{2}(\mathbb{R})$-orbit $\mathrm{SL}_{2}(\mathbb{R}) \cdot(X, q)$ covers a holomorphic and isometric immersion of the hyperbolic plane called a complex geodesic:

$$
f_{(X, q)}: \mathbb{H}=\mathrm{SL}_{2}(\mathbb{R}) / \mathrm{SO}_{2}(\mathbb{R}) \rightarrow \mathcal{M}_{g}
$$

The map $f_{(X, q)}$ further factors through the quotient by the Veech group $\operatorname{SL}(X, q)=$ $\operatorname{Stab}(X, q)$ in $\mathrm{SL}_{2}(\mathbb{R})$ and the projectivizations $\mathbb{P} Q \mathcal{M}_{g}=Q \mathcal{M}_{g} / \mathbb{C}^{*}$ and $\mathbb{P} \Omega \mathcal{M}_{g}=$ $\Omega \mathcal{M}_{g} / \mathbb{C}^{*}$ are foliated by complex geodesics. The $\mathrm{SL}_{2}(\mathbb{R})$-action preserves the quadratic differentials which are squares of holomorphic one-forms. The bundle $\Omega \mathcal{M}_{g}$ extends to the bundle $\Omega \widetilde{\mathcal{M}}_{g}$ whose fiber over the nodal surface with compact Jacobian $X$ with components $X_{i}$ is isomorphic to $\oplus_{i} \Omega\left(X_{i}\right)$. The $\mathrm{SL}_{2}(\mathbb{R})$-action extends naturally to $\Omega \widetilde{\mathcal{M}}_{g}$. The bundle $Q^{G} \mathcal{M}_{g}(G) \rightarrow \mathcal{M}_{g}(G)$ parametrizing $G$-invariant quadratic differentials is closed under the foliation by complex geodesics.

Teichmüller curves. When $\operatorname{SL}(X, q)$ is a lattice, i.e. $\operatorname{SL}(X, q)$ has finite covolume in $\mathrm{SL}_{2}(\mathbb{R})$, the complex geodesic $f_{(X, q)}$ factors through an algebraic and isometric immersion of the quotient $C=\mathbb{H} / \mathrm{SL}(X, q)$. Such an immersion is called a Teichmüller curve. If $P_{i}$ are polygons whose vertices lie in the lattice $\mathbb{Z}[i] \subset \mathbb{C}$, then the quadratic differential $(X, q)=\bigcup_{i}\left(P_{i}, d z^{2}\right) / \sim$ is square-tiled, $\mathrm{SL}(X, q)$ is a lattice in $\mathrm{SL}_{2}(\mathbb{Z})$ and $(X, q)$ generates a Teichmüller curve.

Topological invariants of hyperbolic Riemann surfaces. The topological invariants of a two real dimensional finite volume hyperbolic orbifold $V$ with real dimension two include the number of components $h^{0}(V)$, the genus $g(V)=\operatorname{dim}_{\mathbb{C}} \Omega(V)$, the number of cusps $C(V)$ and the number of orbifold points $e_{n}(V)$ of order $n$ for 
$n>1$. These numbers are combined to give an orbifold Euler characteristic $\chi(V)$ :

$$
\chi(V)=2 h^{0}(V)-2 g(V)-C(V)-\sum_{n} e_{n}(V)\left(1-\frac{1}{n}\right) .
$$

When $V$ is connected (i.e. $h^{0}(V)=1$ ) the numbers $g(V), e_{n}(V)$ and $C(V)$ determine the homeomorphism type of $V$.

\subsection{The Weierstrass curve}

In this section we will collect background related to the Weierstrass curve and its components. References for this section include [Mc1, Mc4, Ba] for the definition of $W_{D}$, [Mc3] for the components of $W_{D}$ and [At] for spin structures on Riemann surfaces.

Eigenforms and the Weierstrass curve. Fix a surface $X \in \widetilde{\mathcal{M}}_{2}$ and a proper $\mathcal{O}_{D}$-action $\iota: \mathcal{O}_{D} \rightarrow \operatorname{End}(\operatorname{Jac}(X))$. The space of one-forms up to scale $\mathbb{P} \Omega(X)$ has a distinguished point corresponding to the line of eigenforms $[\omega]$ with:

$$
\iota(x) \omega=\sigma_{+}(x) \omega
$$

where $\sigma_{+}$is the homomorphism $\mathcal{O}_{D} \rightarrow \mathbb{R}$ with $\sigma_{+}(\sqrt{D})>0$. The map sending $(X, \iota)$ to its $\sigma_{+}$-eigenform up to scale gives an embedding of the Hilbert modular surface $X_{D}$ in $\mathbb{P} \Omega \widetilde{\mathcal{M}}_{2}$ (cf. [Ba], Section 4.3). The images of $X_{D}$ and $X_{E}$ in $\mathbb{P} \Omega \widetilde{\mathcal{M}}_{2}$ are disjoint whenever $D \neq E$.

The Weierstrass curve $W_{D}$ parametrizes the family of surfaces with real multiplication by $\mathcal{O}_{D}$ whose eigenforms have a double zero:

$$
W_{D}=\left\{\begin{array}{c}
X \in \mathcal{M}_{2}, \iota \text { is a proper } \mathcal{O}_{D^{-}} \text {action on } \\
(X, \iota): \quad \operatorname{Jac}(X), \text { and the } \sigma_{+} \text {-eigenform up to } \\
\text { scale }[\omega] \in \mathbb{P} \Omega(X) \text { has a double zero }
\end{array}\right\} / \sim .
$$


The period map embeds $W_{D}$ as a suborbifold of $X_{D}$.

The Hilbert modular surface $X_{D}$ and the Weierstrass curve $W_{D}$ are foliated by Teichmüller geodesics [Ca, Mc1]:

Theorem 2.1 (Calta, McMullen). The images of $W_{D}$ and $X_{D}$ in $\mathbb{P} \Omega \widetilde{\mathcal{M}}_{2}$ are closed under the foliation by complex geodesics.

Orbifolds points on $W_{D}$. Since $W_{D}$ is a suborbifold of $X_{D}$, the orbifold points on $W_{D}$ correspond to pairs $(X, \iota)$ where $\iota: \mathcal{O}_{D} \rightarrow \operatorname{End}(\operatorname{Jac}(X))$ extends to complex multiplication by an order containing $\mathcal{O}_{D}\left[\zeta_{n}\right]$ for some $n>2$. Since the automorphisms of $\operatorname{Jac}(X)$ coincide with automorphisms of $X,(X, \iota)$ is an orbifold point on $W_{D}$ if and only if $X$ has an automorphism commuting with $\iota\left(\mathcal{O}_{D}\right)$. The orbifold order of $(X, \iota)$ is the cardinality of the group $\operatorname{Aut}(X)^{\iota} / \eta$ of automorphisms commuting with $\iota\left(\mathcal{O}_{D}\right)$ modulo the hyperelliptic involution.

Components of $W_{D}$. The components of $W_{D}$ were enumerated in [Mc3]. Usually $W_{D}$ is irreducible; when $W_{D}$ is reducible the components of $W_{D}$ can be distinguished by a spin invariant which we describe below.

A spin structure on a topological surface $X$ is a quadratic form

$$
q: H_{1}(X, \mathbb{Z} / 2 \mathbb{Z}) \rightarrow \mathbb{Z} / 2 \mathbb{Z}
$$

satisfying $q(x+y)=q(x)+q(y)+\langle x, y\rangle$ where $\langle$,$\rangle is the intersection pairing. The$ parity of $q$ is given by the Arf-invariant:

$$
\operatorname{Arf}(q)=\sum_{i} q\left(A_{i}\right) q\left(B_{i}\right) \in \mathbb{Z} / 2 \mathbb{Z}
$$

for a standard symplectic basis $\left\{A_{i}, B_{i}\right\}$ of $H_{1}(X, \mathbb{Z} / 2 \mathbb{Z})$. The number $\operatorname{Arf}(q)$ is independent of basis.

If $X \in \mathcal{M}_{2}$ is a Riemann surface, a one-form $\omega \in \Omega(X)$ with double zero determines an odd spin structure $q$, i.e. $\operatorname{Arf}(q)=1$. Any $x \in H_{1}(X, \mathbb{Z} / 2 \mathbb{Z})$ is the image of the fundamental class of the circle $S^{1}$ under a smooth immersion $C: S^{1} \rightarrow X$ that 
avoids the zero $Z(\omega)$ of $\omega$. The Gauss map is the map $S^{1} \rightarrow S^{1}$ given by:

$$
G_{x}: \theta \mapsto \frac{\omega\left(C^{\prime}(\theta)\right)}{\left|\omega\left(C^{\prime}(\theta)\right)\right|},
$$

and the function $q(x)=\operatorname{deg} G_{x}+1 \bmod 2$ does not depend on the immersion $C$ and defines an odd spin structure on $X$.

Now fix $D>1$ with $D \equiv 1 \bmod 8$ and a point $(X, \iota) \in W_{D}$ and let $f$ be the conductor of $\mathcal{O}_{D}$. Let $q$ be the spin structure defined by the $\sigma_{+}$-eigenform up to scale $[\omega]$. The Jacobian endomorphism $(f+\sqrt{D}) / 2$ gives a linear transformation on $H_{1}(X, \mathbb{Z} / 2 \mathbb{Z})$ whose image $V$ is a two dimensional subspace. The spin invariant of $(X, \iota)$ is defined to be the Arf-invariant of $q$ restricted to $V$ :

$$
\epsilon(X, \omega)=\epsilon(X, \iota)=\operatorname{Arf}\left(\left.q\right|_{V}\right) .
$$

The spin component associated to $\epsilon \in \mathbb{Z} / 2 \mathbb{Z}$ is given by:

$$
W_{D}^{\epsilon}=\left\{(X, \iota):(X, \iota) \in W_{D} \text { and } \epsilon(X, \iota)=\epsilon\right\}
$$

McMullen showed that the discriminant for real multiplication and the spin invariant distinguish the irreducible components of $\bigcup_{D} W_{D}$ ([Mc3], Thm. 1.1):

Theorem 2.2 (McMullen). The curves $W_{1}$ and $W_{4}$ are empty. For discriminants $D>9$ with $D \equiv 1 \bmod 8$, the spin components $W_{D}^{0}$ and $W_{D}^{1}$ are non-empty and irreducible. For all other discriminants, $W_{D}$ is irreducible.

Bouw and Möller showed the spin components are Galois conjugate and homeomorphic ([BM1], Thm. 3.3):

Theorem 2.3 (Bouw-Möller). Fix $D \equiv 1 \bmod 8$ with $D$ not a square. The curves $W_{D}^{0}$ and $W_{D}^{1}$ have algebraic models defined over $\mathbb{Q}(\sqrt{D})$ and are Galois conjugate.

When $D=f^{2}$ is an odd square, the spin invariant has a more elementary interpretation. For such discriminants, $(X, \iota) \in W_{D}$ is a degree $f$ branched cover of an 
elliptic curve $E$ branched over a single point $B$, and the number $N=\left|f^{-1}(B) \cap X^{W}\right|$ determines the spin invariant $\epsilon(X, \iota)=\frac{N-1}{2}$. 


\section{Chapter 3}

\section{Orbifold points on Hilbert modular surfaces}

In this section, we will show that most of the orbifold points on $\bigcup_{D} X_{D}$ are Jacobians of $D_{8^{-}}$and $D_{12^{-}}$-surfaces with complex multiplication. In $\S 4,5$ and A.1 we will show that every complex multiplication point on $\mathcal{M}_{2}\left(D_{8}\right)$ and $\mathcal{M}_{2}\left(D_{12}\right)$ gives orbifold point on $\bigcup_{D} X_{D}$ establishing Theorem 1.5.

Automorphisms of abelian surfaces. Let $\widetilde{S}_{4}$ and $\widetilde{D}_{12}$ be the automorphism groups of the polarized abelian varieties $A_{\sqrt{-2} / 2}$ and $\widetilde{A}_{\sqrt{-3} / 3}$ defined in Sections 1 and A.1. The groups $\widetilde{S}_{4}$ and $\widetilde{D}_{12}$ are $\mathbb{Z} / 2 \mathbb{Z}$-central extensions of the groups $S_{4}$ and $D_{12}$ respectively. The following proposition is well known (cf. [BL], pg. 340):

Proposition 3.1. The automorphism group of a two dimensional principally polarized abelian variety which is not a product of elliptic curves is isomorphic to one of the following: $\mathbb{Z} / 2 \mathbb{Z}, \mathbb{Z} / 10 \mathbb{Z}, D_{4}, D_{8}, D_{12}, \widetilde{S}_{4}$ or $\widetilde{D}_{12}$.

Note that the group $\widetilde{S}_{4}$ contains $D_{8}$ and the group $\widetilde{D}_{12}$ contains both $D_{8}$ and $D_{12}$.

Orbifold points on Hilbert modular surfaces. We will prove the following variant of Proposition 3.1:

Proposition 3.2. Fix a discriminant $D>0$, an integer $n>2$ and an abelian variety $B \in A_{2}$ with $\mathcal{O}_{D}\left[\zeta_{n}\right]$-action $\iota$. One of the following holds: 
- $B$ is a product of elliptic curves,

- $(B, \iota)$ is a point of orbifold order five on $X_{5}$,

- An iterate of $\iota\left(\zeta_{n}\right)$ extends to a faithful action of $D_{8}$ on $B$ by automorphisms, or

- An iterate of $\iota\left(\zeta_{n}\right)$ extends to a faithful action of $D_{12}$ on $B$ by automorphisms.

Recall that the orbifold points of order $n$ on $X_{D}$ correspond to abelian varieties with complex multiplication by orders containing $\mathcal{O}_{D}\left[\zeta_{2 n}\right]$ (§2.1). Proposition 3.2 shows that the orbifold points on $\bigcup_{D} X_{D}$ which are not products of elliptic curves are the points of order five on $X_{5}$ and Jacobians of $D_{8}$ - and $D_{12}$-surfaces with complex multiplication.

Proof. Every two dimensional principally polarized abelian variety is a product of elliptic curves or the Jacobian of a smooth surface (cf. 2.2). Suppose $B$ is the Jacobian of $X \in \mathcal{M}_{2}$. Let $\phi$ be the automorphism of $X$ inducing $\iota\left(\zeta_{n}\right)$ on $\operatorname{Jac}(X)$, and let $\sigma_{\phi}$ be the induced permutation of the Weierstrass points $X^{W}=\operatorname{Fix}(\eta)$. Since $\phi$ and each of its iterates can fix at most three points on the sphere $X / \eta$, the conjugacy class of $\sigma_{\phi}$ is one of the following:

- [2,2,2]: There is a coordinate $x: X / \eta \stackrel{\sim}{\rightarrow} \widehat{\mathbb{C}}$ with $x \circ \phi=-x$ and $x\left(X^{W}\right)=$ $\{ \pm 1, \pm a, \pm b\}$ for some $a$ and $b \in \mathbb{C}^{*}$. The field of functions on $X$ satisfies:

$$
\mathbb{C}(X) \cong \frac{\mathbb{C}(z)[x]}{\left(z^{2}-\left(x^{2}-1\right)\left(x^{2}-a^{2}\right)\left(x^{2}-b^{2}\right)\right)},
$$

and $\phi(z, x)=(z,-x)$ or $(-z,-x)$. This is impossible since $\phi^{2} \neq 1$.

- [2,2,1,1]: Let $P_{1}, P_{2}, Q_{1}$ and $Q_{2}$ be Weierstrass points so $\phi\left(P_{i}\right)=P_{i}$ and $X^{W}=\left\{P_{1}, P_{2}, Q_{1}, \phi\left(Q_{1}\right), Q_{2}, \phi\left(Q_{2}\right)\right\}$. There is a unique coordinate $x: X / \eta \rightarrow$ $\widehat{\mathbb{C}}$ with $x\left(P_{1}\right)=1, x\left(P_{2}\right)=-1$ and $x\left(Q_{1}\right)=-x\left(Q_{2}\right)$. This coordinate satisfies $x \circ \phi=1 / x$ and $x\left(X^{W}\right)=\left\{ \pm 1, \pm x\left(Q_{1}\right), \pm x\left(Q_{1}\right)^{-1}\right\}$. The field of functions on $X$ satisfies:

$$
\mathbb{C}(X) \cong \frac{\mathbb{C}(z)[x]}{\left(z^{2}-\left(x^{2}-1\right)\left(x^{4}-a x^{2}+1\right)\right)},
$$


where $a=x\left(Q_{1}\right)^{2}+x\left(Q_{1}\right)^{-2}$. The surface $X$ has a faithful $D_{8}$-action generated by $r(z, x)=(z,-x)$ and $J=\left(i z / x^{3}, 1 / x\right)$. Since $x \circ \phi=1 / x, \phi=J$ or $J^{3}$ and $\iota\left(\zeta_{n}\right)$ extends to a faithful action of $D_{8}$ on $B$.

- [3,3]: Let $P$ and $Q$ be points in $X^{W}$ in different $\phi$-orbits. There is a coordinate $x: X / \eta \stackrel{\sim}{\rightarrow} \widehat{\mathbb{C}}$ with $x \circ \phi=\zeta_{3} x$ and $x(P)=x(Q)^{-1}$. Setting $a=x(P)$, we have $x\left(X^{W}\right)=\left\{a, \zeta_{3} a, \zeta_{3}^{2} a, a^{-1}, \zeta_{3} a^{-1}, \zeta_{3} a^{-2}\right\}$ and the field of functions on $X$ satisfies:

$$
\mathbb{C}(X) \cong \frac{\mathbb{C}(z)[x]}{\left(z^{2}-\left(x^{3}-a^{3}\right)\left(x^{3}-a^{-3}\right)\right)} .
$$

The surface $X$ has a faithful $D_{12}$-action generated by $r(z, x)=\left(z / x^{3}, 1 / x\right)$ and $Z(z, x)=\left(-z, \zeta_{3} x\right)$. Since $x \circ \phi=\zeta_{3} x, \phi$ is in the subgroup of $D_{12}$ generated by $Z$ and $\iota\left(\zeta_{n}\right)$ extends to an action of $D_{12}$ on $B$.

- $[4,1,1]$ or $[4,2]:$ The automorphism $\phi^{2}$ acts on $X^{W}$ by a permutation in the conjugacy class $[2,2,1,1]$ and $B$ has a faithful $D_{8}$-action by automorphisms extending $\iota\left(\zeta_{n}^{2}\right)$. The surface $X$ is the unique genus two surface whose automorphism group is $\widetilde{S}_{4}$ :

$$
\mathbb{C}(X) \cong \frac{\mathbb{C}(z)[x]}{\left(z^{2}-\left(x^{2}-1\right)\left(x^{4}+6 x^{2}+1\right)\right)}
$$

- [5,1]: There is a coordinate $x: X / \eta \stackrel{\sim}{\rightarrow} \widehat{\mathbb{C}}$ with $x \circ \phi=\zeta_{5} x$ and $x\left(X^{W}\right)=$ $\left\{1, \zeta_{5}^{ \pm 1}, \zeta_{5}^{ \pm 2}, \infty\right\}$. The surface $X$ is the unique genus two surface with order five automorphism and satisfies:

$$
\mathbb{C}(X) \cong \frac{\mathbb{C}(z)[x]}{\left(z^{2}-\left(x^{5}-1\right)\right)}
$$

The order for real multiplication $\mathcal{O}_{D}$ is $\mathcal{O}_{5}=\mathbb{Z}\left[\zeta_{5}\right] \cap \mathbb{R}$.

- [6]: The automorphism $\phi^{2}$ induces a permutation of $X^{W}$ in the conjugacy class $[3,3]$ and $B$ has a faithful $D_{12}$-action extending $\iota\left(\zeta_{n}^{2}\right)$. The surface $X$ is the 
unique genus two surface whose automorphism group is $\widetilde{D}_{12}$ and $X$ satisfies:

$$
\mathbb{C}(X) \cong \frac{\mathbb{C}(z)[x]}{\left(z^{2}-\left(x^{6}+1\right)\right)}
$$

We record the following consequence of the proof of Proposition 3.2 which will be useful in our analysis of the $D_{8}$-family:

Proposition 3.3. Suppose $X \in \mathcal{M}_{2}$ has an order four automorphism $\phi$. The induced permutation $\sigma_{\phi} \in \operatorname{Sym}\left(X^{W}\right)$ is in the conjugacy class $[2,2,1,1]$ and $\phi$ extends to an action of $D_{8}$.

Proof. Since the kernel of $\operatorname{Aut}(X) \rightarrow \operatorname{Sym}\left(X^{W}\right)$ is generated by the hyperelliptic involution, the permutation $\sigma_{\phi}$ has order two or four. Since the automorphisms inducing permutations in the conjugacy class $[2,2,2]$ have order two and those inducing permutations in $[4,1,1]$ and $[4,2]$ have order eight, the permutation $\sigma_{\phi}$ must be in $[2,2,1,1]$.

Orbifold points on $W_{D}$. Our characterization of the orbifold points on $X_{D}$ which lie on $W_{D}$ follows readily from Proposition 3.2.

Proposition 3.4. Fix $X \in \mathcal{M}_{2}$ with $\mathcal{O}_{D}\left[\zeta_{n}\right]$-action $\iota$ on $\operatorname{Jac}(X)$ and suppose an eigenform for $\iota$ has a double zero. Either:

- $X$ is the genus two surface with order five automorphism, or

- $X$ admits a faithful action of $D_{8}$ extending an iterate of $\iota\left(\zeta_{n}\right)$.

Proof. If $\phi$ is the automorphism inducing $\iota\left(\zeta_{n}\right)$ on $\operatorname{Jac}(X)$ and $\sigma_{\phi}$ is the induced permutation on $X^{W}$, the conjugacy class of $\sigma_{\phi}$ in $\operatorname{Sym}\left(X^{W}\right)$ is $[2,2,1,1],[4,1,1]$ or $[5,1]$ since $\phi$ fixes at least one point in $X^{W}$, namely the zero of the eigenform.

Remark. The orbifold points on $X_{D}$ which are products of elliptic curves are also easy to characterize. For $D=1$, the complex orbifold $X_{1}$ is isomorphic to the product 
$\mathcal{M}_{1} \times \mathcal{M}_{1}$ and the product $E \times F$ with $\mathcal{O}_{1}$ generated by $\left(\begin{array}{ll}1 & 0 \\ 0 & 0\end{array}\right)$ is an orbifold point if and only if $E$ or $F$ is an orbifold point on $\mathcal{M}_{1}$. For $D \geq 4$, if the product $E \times F$ is labeled by an orbifold point on $X_{D}$, then $E$ and $F$ are isomorphic and have complex multiplication by $\mathbb{Z}\left[\zeta_{3}\right], \mathbb{Z}[i]$ or an order in $\mathbb{Q}(\sqrt{-D})$. 


\section{Chapter 4}

\section{The $D_{8}$-family}

The family $\widetilde{\mathcal{M}}_{2}\left(D_{8}\right)$ parametrizes pairs $(X, \rho)$ where $X$ is in $\widetilde{\mathcal{M}}_{2}$ and $\rho: D_{8} \rightarrow$ $\operatorname{Aut}(X)$ is an injective homomorphism (cf. §2.2). In this section, we will identify the components of $\widetilde{\mathcal{M}}_{2}\left(D_{8}\right)$ and study the component whose general member is smooth. For $(X, \rho) \in \mathcal{M}_{2}\left(D_{8}\right)$ we will show that the correspondence $X \rightarrow X / r \times X / J r$ induces a degree two isogeny between elliptic curves:

$$
c_{\rho}: \operatorname{Jac}(X / r) \rightarrow \operatorname{Jac}(X / J r)
$$

We will compute $\operatorname{Jac}(X)$ and $\mathbb{C}(X)$ in terms of $c_{\rho}$ to show:

Proposition 4.1. The map $(X, \rho) \mapsto c_{\rho}$ gives an embedding:

$$
\mathcal{M}_{2}\left(D_{8}\right) \rightarrow Y_{0}(2)
$$

whose image is the complement of the degree two endomorphism of the square torus. The action of the non-trivial outer automorphism $\sigma$ of $D_{8}$ on $\mathcal{M}_{2}\left(D_{8}\right)$ is induced by the Atkin-Lehner involution on $Y_{0}(2)$.

In $\S 1$, we defined the domain $U$, the field $K_{a}$, the abelian variety $A_{\tau}$, the surfaces $X_{\tau}$ and $Y_{w}$ and the functions $a(\tau)$ and $w(\tau)$. We will also show that the surfaces $X_{\tau}$ and $Y_{w(\tau)}$ admit faithful $D_{8}$-actions inducing the isogeny $i_{\tau}$ on $E_{\tau}=\mathbb{C} / \mathbb{Z} \oplus \tau \mathbb{Z}$ whose kernel is generated by the image $T_{\tau}$ of $1 / 2$ in $\mathbb{C} / \mathbb{Z} \oplus \tau \mathbb{Z}$ (see Figure 4-1), establishing: 


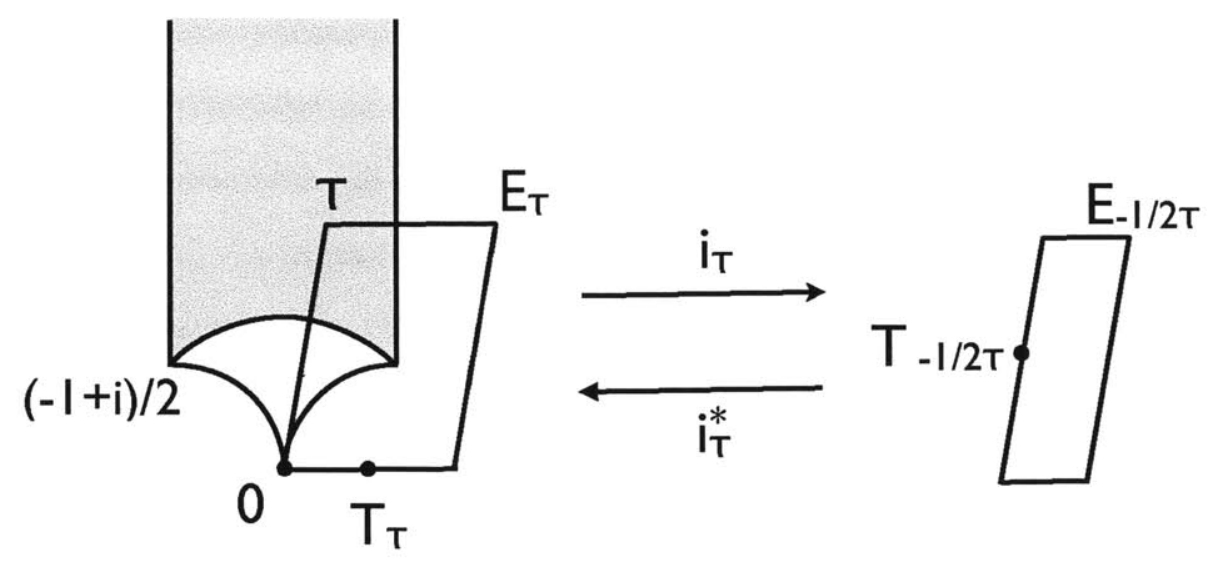

Figure 4-1: The domain $V$ obtained from $U$ and the image of $U$ under $\tau \mapsto-1 / 2 \tau$ is a fundamental domain for the group $\Gamma_{0}(2)$. A point $\tau \in V$ determines an elliptic curve $E_{\tau}=$ $\mathbb{C} / \mathbb{Z} \oplus \tau \mathbb{Z}$ and a degree two isogeny $i_{\tau}$ whose kernel is generated by the point of order two $T_{\tau}$. The isogeny $i_{-1 / 2 \tau}$ is dual to $i_{\tau}$ since $i_{-1 / 2 \tau} \circ i_{\tau}$ is the multiplication by two map on $E_{\tau}$.

Proposition 4.2. Fix $\tau \in U$. The surface $X_{\tau}$ admits a faithful $D_{8}$-action $\rho$ with $c_{\rho}=i_{\tau}$, is isomorphic to the surface $Y_{w(\tau)}$ and satisfies:

$$
\mathbb{C}\left(X_{\tau}\right) \cong K_{a(\tau)} \text { and } \operatorname{Jac}\left(X_{\tau}\right) \cong A_{\tau}
$$

Components of $\widetilde{\mathcal{M}}_{2}\left(D_{8}\right)$. Fix a surface $X \in \widetilde{\mathcal{M}}_{2}$ and let $\rho: D_{8} \rightarrow \operatorname{Aut}(X)$ be a faithful representation of $D_{8}$. We will call $\rho$ proper if the induced actions of $\mathbb{Z}[r]$ and $\mathbb{Z}[J r]$ on $\operatorname{Jac}(X)$ are proper. We will call $\rho$ improper if it is not proper. We will denote the corresponding components of $\widetilde{\mathcal{M}}_{2}\left(D_{8}\right)$ by $\widetilde{\mathcal{M}}_{2}\left(D_{8}\right)^{p r}$ and $\widetilde{\mathcal{M}}_{2}\left(D_{8}\right)^{i m}$.

A faithful representation on a smooth surface is automatically proper and we will show that $\widetilde{\mathcal{M}}_{2}\left(D_{8}\right)^{p r}$ is the closure of $\mathcal{M}_{2}\left(D_{8}\right)$. For a nodal surface $X=E \vee F$, an action of $D_{8}$ is proper if and only if both $r$ and $J r$ interchange the components of $X$. For any genus one surface $E \in \mathcal{M}_{1}$, the singular surface $E \vee E$ has a faithful $D_{8}$-action which is improper.

Nodal surfaces. We now characterize the nodal surfaces in $\widetilde{\mathcal{M}}_{2}\left(D_{8}\right)$.

Proposition 4.3. The nodal surface $X=E \vee F$ admits an improper $D_{8}$-action iff $E$ is isomorphic to $F$. The nodal surface $X=E \vee F$ admits a proper $D_{8}$-action if and 
only if both $E$ and $F$ are isomorphic to the square torus.

Proof. It is easy to show that $E \vee E$ has an improper action of $D_{8}$ for any $E \in \mathcal{M}_{1}$. It is also easy to check that when $E=\mathbb{C} / \mathbb{Z}[i]$, the automorphism group of $E \vee E$ contains a proper action of $D_{8}$, which is unique up to conjugation in $\operatorname{Aut}(E \vee E)$.

For the converse, suppose $\rho: D_{8} \rightarrow \operatorname{Aut}(X)$ is injective. Since the subgroup of $\operatorname{Aut}(X)$ fixing the components of $X$ is the commutative group $\operatorname{Aut}(E) \times \operatorname{Aut}(F)$, either $r$ or $J r$ interchanges the components of $X$ and $E$ is isomorphic to $F$. Now suppose $\rho$ is also proper. Both $r$ and $J r$ interchange the components of $X$ and the composition $J=(J r)(r)$ fixes the components of $X$. Since $J^{4}=1$, both $E$ and $F$ are isomorphic to the square torus.

Remark. Proposition 4.3 can be used to show that $\widetilde{\mathcal{M}}_{2}\left(D_{8}\right)^{i m}$ has two components both of which are isomorphic to $\mathcal{M}_{\mathbf{1}}$ (the components are distinguished by whether $r$ or $J r$ generates a proper $\mathcal{O}_{4}$-action). Also, the proper $D_{8}$-action on $E \vee E$ when $E=\mathbb{C} / \mathbb{Z}[i]$ is unique up to $\sim$ since it is unique up to conjugation in $\operatorname{Aut}(E \vee E)$.

Weierstrass points. Let $\phi: D_{8} \rightarrow S_{6}$ be the representation into the symmetric group on six letters given by:

$$
\phi(r)=(12)(34)(56) \text { and } \phi(J)=(13)(24) .
$$

For a proper $D_{8}$-surface, the action $D_{8}$ induces on the Weierstrass points is isomorphic to $\phi$.

Proposition 4.4. Fix a surface $X \in \widetilde{\mathcal{M}}_{2}$ with a proper $D_{8}$-action $\rho$. There is a bijection $X^{W} \rightarrow S_{6} / S_{5}$ inducing a homomorphism $f: \operatorname{Sym}\left(X^{W}\right) \rightarrow S_{6}$ so the following diagram commutes:

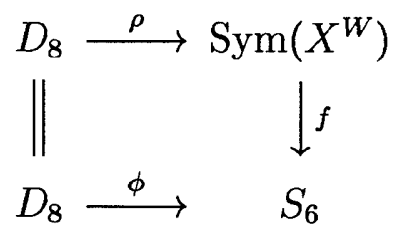


Proof. We will show that $J^{2}$ is the hyperelliptic involution and that the involutions $r$ and $J r$ fix no point in $X^{W}$. As a consequence, the action $D_{8}$ induces on $X^{W}$ factors through the commutative group $D_{4} \cong D_{8} / J^{2}$ and the proposition follows easily.

Since $\mathbb{Z}[r]$ and $\mathbb{Z}[J r]$ act faithfully on $\operatorname{Jac}(X)$, the eigenspaces for $r$ and $J r$ in $\Omega(X)$ are one-dimensional. Since $r$ and $J r$ do not commute, their eigenspaces are distinct. The automorphism $J^{2}$ commutes with both $r$ and $J r$ and fixes every line in $\Omega(X)$. The automorphism $J^{2}$ is the hyperelliptic involution.

Since $\mathbb{Z}[r]$ and $\mathbb{Z}[J r]$ are proper, the quotients $X / r$ and $X / J r$ have genus one. For smooth $X$, the fixed points of $r$ and $J r$ are the simple zeros of the one-forms pulled back from $X / r$ and $X / J r$, so they are disjoint from $X^{W}$. For nodal $X, r$ and $J r$ interchange the components of $X$ and again the fixed points of $r$ and $J r$ are disjoint from $X^{W}$.

We will denote by $\operatorname{Fix}_{W}(J)$ the set of Weierstrass points fixed by $J$. We will need the following fact in our discussion of surfaces obtained from polygons:

Proposition 4.5. Two proper $D_{8}$-surfaces $\left(X_{1}, \rho_{1}\right)$ and $\left(X_{2}, \rho_{2}\right)$ have an isomorphism $\psi: X_{1} \rightarrow X_{2}$ intertwining $\rho_{i}(J)$ if and only if:

$$
\left(X_{1}, \rho_{1}\right) \sim\left(X_{2}, \rho_{2}\right) \text { or }\left(X_{1}, \rho_{1}\right) \sim\left(X_{2}, \rho_{2} \circ \sigma\right) .
$$

Proof. Let $t$ be the automorphism of $X_{1}$ pulled back from $\rho_{2}(r)$ under $\psi$. By Proposition 4.4, the action of $t$ on $X_{1}^{W}$ coincides with that of $r$ or $J r$ and, as a consequence, $t$ is one of $r, J r, \eta r$ or $\eta J r$. If $t$ is $r$ or $J r, \phi$ gives the desired equivalence. Otherwise, $\phi \circ \rho_{1}(J)$ gives the desired equivalence.

Proper $D_{8}$-actions, correspondences and isogeny. We will now show that a proper $D_{8}$-surface $(X, \rho) \in \widetilde{\mathcal{M}}_{2}\left(D_{8}\right)^{p r}$ determines a degree two isogeny $c_{\rho}$ between the genus one surfaces $\operatorname{Jac}(X / r)$ and $\operatorname{Jac}(X / J r)$. The non-trivial outer automorphism $\sigma$ of $D_{8}$ has $\sigma(r)=J r$ and $\sigma(J)=J$. We will also show that the surface $(X, \rho \circ \sigma)$ determines the dual isogeny $c_{\rho}^{*}$. 
Recall that the quotients $E=X / r$ and $F=X / J r$ have genus one, elliptic involutions $\eta_{E}$ and $\eta_{F}$ induced by $\eta$, natural base points $E[1]=\operatorname{Fix}(\eta r) / r$ and $F[1]=\operatorname{Fix}(r) / \eta r$ and identifications $E[2]^{*}=X^{W} / r$ and $F[2]^{*}=X^{W} / J r$ (see $\S 2.2$ ).

Proposition 4.6. Suppose $X \in \widetilde{\mathcal{M}}_{2}$ has a proper $D_{8}$-action $\rho$. The correspondence $X \rightarrow X / r \times X / J r$ induces an isogeny:

$$
c_{\rho}: \operatorname{Jac}(X / r) \rightarrow \operatorname{Jac}(X / J r)
$$

whose kernel is generated by the point of order two $\left[\operatorname{Fix}_{W}(J) / r-\operatorname{Fix}(\eta r) / r\right]$. The isogeny $c_{\rho}$ is dual to the isogeny $c_{\rho \circ \sigma}$.

Proof. We will show that $\operatorname{ker}\left(c_{\rho}\right)$ contains the point of order two labeled by $\operatorname{Fix}_{W}(J) / r$ and that $c_{\rho} \circ c_{\rho \circ \sigma}$ is the multiplication by two map on $\operatorname{Jac}(X / r)$. It follows that both $c_{\rho}$ and $c_{\rho \circ \sigma}$ have degree two and $c_{\rho}^{*}=c_{\rho \circ \sigma}$.

The isogeny $c_{\rho}$ is given by summing the images of the fibers of the map $X \rightarrow X / r$ under the map $X \rightarrow X / J r$. For the point in $X / r$ labeled by the $r$-orbit $\{Q, r Q\}$ we have:

$$
c_{\rho}(\{Q, r Q\})=\{Q, J r Q\}+{ }_{F}\{r Q, J Q\} .
$$

The point $\operatorname{Fix}_{W}(J) / r$ is in $\operatorname{ker}\left(c_{\rho}\right)$ since $\{r Q, J Q\}=\eta_{F}(\{Q, J r Q\})$ whenever $J(Q)=$ $Q$.

The non-trivial outer automorphism $\sigma$ of $D_{8}$ is generated by $\sigma(J)=J$ and $\sigma(r)=$ $J r$. Using the formula above for $c_{\rho}$ and the relations on $D_{8}$, we have that $c_{\rho \circ \sigma}$ 。 $c_{\rho}(\{Q, r Q\})$ is equal to:

$$
\{Q, r Q\}+{ }_{E}\left\{J r Q, J^{3} Q\right\}+_{E}\{r Q, Q\}+_{E}\{J Q, r J Q\}
$$

Since $J^{2}=\eta$, we have $\left\{J r Q, J^{3} Q\right\}=\eta_{E}(\{J Q, r J Q\})$ and the second and fourth terms cancel, giving $c_{\rho} \circ c_{\rho \circ \sigma}(\{Q, r Q\})=\{Q, r Q\}+{ }_{E}\{Q, r Q\}$.

The unique nodal and proper $D_{8}$-surface $(X, \rho)$ has:

$$
\operatorname{Jac}(X / r)=\operatorname{Jac}(X / J r)=\mathbb{C} / \mathbb{Z}[i]
$$


and $c_{\rho}=i_{(1+i) / 2}$ is point of orbifold order two on the modular curve $Y_{0}(2)$ (see $\S 2.1$ ).

Jacobians. We now compute the Jacobian $\operatorname{Jac}(X)$ for a proper $D_{8}$-surface $(X, \rho)$ in terms of the isogeny $c_{\rho}$.

Proposition 4.7. Fix a $D_{8}$-surface $(X, \rho) \in \widetilde{\mathcal{M}}_{2}\left(D_{8}\right)^{\text {pr }}$ and a number $\tau \in \mathbb{H}$ so $c_{\rho}=i_{\tau}$. The Jacobian of $X$ satisfies:

$$
\operatorname{Jac}(X) \cong A_{\tau}
$$

The polarized abelian variety $A_{\tau}$ was defined in $\S 1$ and the isogeny $i_{\tau}$ is the map on $E_{\tau}=\mathbb{C} / \mathbb{Z} \oplus \tau \mathbb{Z}$ whose kernel is generated by the image $T_{\tau}$ of $1 / 2$ in $\mathbb{C} / \mathbb{Z} \oplus \tau \mathbb{Z}$.

Proof. Since the lattice $\Lambda_{\tau}$ contains the lattice $\mathbb{Z}\left\langle\left(\begin{array}{l}2 \\ 0\end{array}\right),\left(\begin{array}{c}2 \tau \\ 0\end{array}\right),\left(\begin{array}{c}0 \\ 2\end{array}\right),\left(\begin{array}{c}0 \\ 2 \tau\end{array}\right)\right\rangle$, the abelian variety $A_{\tau}$ satisfies:

$$
A_{\tau} \cong\left(E_{\tau} \times E_{\tau}\right) / \Gamma^{T_{\tau}}
$$

where $\Gamma^{T_{\tau}} \subset E_{\tau}[2]^{*} \times E_{\tau}[2]^{*}$ is the graph of the transposition fixing $T_{\tau}$. Twice the product polarization on $E_{\tau} \times E_{\tau}$ is pulled back from the principal polarization on $A_{\tau}$ under the isogeny $E_{\tau} \times E_{\tau} \rightarrow A_{\tau}$.

Let $E=\operatorname{Jac}(X / r)$ and $\widetilde{E}=\operatorname{Jac}(X / \eta r)$. Since $c_{\rho}=i_{\tau}$, the elliptic curve $E_{\tau}$ is isomorphic to $E$. The automorphism $J$ induces an isomorphism:

$$
\phi: X / r \stackrel{\sim}{\rightarrow} X / \eta r
$$

since $J^{-1} r J=\eta r$ so $\widetilde{E}$ is also isomorphic to $E_{\tau}$. The equality $X^{W} / r=X^{W} / \eta r$ gives a bijection on the points of order two $\psi: E[2]^{*} \rightarrow \widetilde{E}[2]^{*}$ and the composition $\phi^{-1} \circ \psi$ is the action $J$ induces on $X^{W} / r=E[2]^{*}$. We computed the action of $\rho$ on $X^{W}$ in Proposition 4.4 and $\phi^{-1} \circ \psi$ is the transposition fixing $T_{\tau}$.

The map $X \rightarrow X / r \times X / \eta r$ factors through a degree four map on Jacobians $\operatorname{Jac}(X) \rightarrow E \times \widetilde{E}$. The image of $X^{W}$ in $E \times \widetilde{E} \cong E_{\tau} \times E_{\tau}$ is the graph $\Gamma^{T_{\tau}}$ of $\psi^{-1} \circ \phi$ and the image of $\operatorname{Jac}(X)[2]$ in $E_{\tau} \times E_{\tau}$ is the subgroup generated by $\Gamma^{T_{\tau}}$. The quotient 
map:

$$
\operatorname{Jac}(X) \rightarrow\left(E_{\tau} \times E_{\tau}\right) / \Gamma^{T_{\tau}}
$$

has degree sixteen, vanishes on the two torsion $\operatorname{Jac}(X)[2]$ and factors through multiplication by two to give an isomorphism. The polarization is a product since the self-adjoint operator $r$ has eigenspaces pulled back from $\Omega(E)$ and $\Omega(\widetilde{E})$. Unimodularity implies the polarization is twice the product polarization.

Remark. More generally, if $Y \in \widetilde{M}_{2}$ has an involution $r$ generating a proper $\mathcal{O}_{4^{-}}$ action on $\operatorname{Jac}(Y)$, the quotients $E_{1}=Y / r$ and $E_{2}=Y / \eta r$ have genus one, natural elliptic involutions and base points. The points of order two on $E_{1}$ and $E_{2}$ have an identification coming from $Y^{W}$ :

$$
E_{1}[2]^{*}=Y^{W} / r=Y^{W} / \eta r=E_{2}[2]^{*}
$$

The middle equality comes from the fact that $\eta$ fixes $Y^{W}$ pointwise. The Jacobian of $Y$ satisfies:

$$
\operatorname{Jac}(Y) \cong\left(E_{1} \times E_{2}\right) / \Gamma^{W}
$$

where $\Gamma^{W}$ is the graph of this identification and the right hand side is polarized by twice the product polarization. Proposition 4.7 is a special instance of this computation.

Since the Jacobian of a $D_{8}$-surface can be computed from the isogeny $c_{\rho}$, we have established that the map $(X, \rho) \mapsto c_{\rho}$ embeds $\mathcal{M}_{2}\left(D_{8}\right)$ in $Y_{0}(2)$ :

Proof of Proposition 4.1. We will show that the map $\widetilde{\mathcal{M}}_{2}\left(D_{8}\right)^{p r} \rightarrow Y_{0}(2)$ given by $(X, \rho) \mapsto c_{\rho}$ is an isomorphism. The remaining claims follow from Proposition 4.6 and our observation that the unique nodal and proper $D_{8}$-surface maps to the isogeny $i_{(1+i) / 2}$.

We will define an inverse for the map $(X, \rho) \mapsto c_{\rho}$. Fix an isogeny $i_{\tau}$ in $Y_{0}(2)$ and let $X$ be the (possibly nodal) surface with Jacobian $A_{\tau}$. Let $\rho_{\tau}: D_{8} \rightarrow \operatorname{Aut}(X)$ be the action of $D_{8}$ inducing the Jacobian automorphisms $\rho_{\tau}(r)=\left(\begin{array}{cc}1 & 0 \\ 0 & -1\end{array}\right)$ and $\rho_{\tau}(J)=\left(\begin{array}{cc}0 & 1 \\ -1 & 0\end{array}\right)$. The map $i_{\tau} \rightarrow\left(X_{\tau}, \rho_{\tau}\right)$ is the desired inverse by Proposition 4.7. 
Remark. The family $\widetilde{\mathcal{M}}_{2}\left(D_{8}\right)^{p r}$ is connected and one dimensional. Since there is a unique nodal and proper $D_{8}$-surface, $\widetilde{\mathcal{M}}_{2}\left(D_{8}\right)^{p r}$ is the closure of $\mathcal{M}_{2}\left(D_{8}\right)$ in $\widetilde{\mathcal{M}}_{2}\left(D_{8}\right)$.

Algebraic curves. The function $a(\tau)=-2+\frac{1}{\lambda(\tau) \lambda(\tau+1)}$ defined in $\S 1$ is modular for $\Gamma_{0}(2)$ and covers an isomorphism $a: Y_{0}(2) \rightarrow \mathbb{C} \backslash\{-2, \infty\}$. Using $\lambda\left(\frac{-1+i}{2}\right)=$ $\lambda\left(\frac{1+i}{2}\right)=\frac{1}{2}$, the isomorphism $a$ is uniquely determined by:

$$
a\left(\Gamma_{0}(2) \cdot 0\right)=\infty, a\left(\Gamma_{0}(2) \cdot \infty\right)=-2, \text { and } a\left(\Gamma_{0}(2) \cdot(1+i) / 2\right)=2
$$

The number $a(\tau)$ is characterized by:

$$
\mathbb{C}\left(E_{\tau}\right) \cong \frac{\mathbb{C}(z)[w]}{\left(z^{2}-(w-1)\left(w^{2}-a(\tau) w+1\right)\right)} \text { with } T_{\tau}=\left[w^{-1}(1)-w^{-1}(\infty)\right]
$$

where as usual $E_{\tau}=\mathbb{C} / \mathbb{Z} \oplus \tau \mathbb{Z}$ and $T_{\tau}=\frac{1}{2}+\mathbb{Z} \oplus \tau \mathbb{Z}$.

Proposition 4.8. Fix a smooth $D_{8}$-surface $(X, \rho)$ and $\tau \in \mathbb{H}$ with $c_{\rho}=i_{\tau}$. The field of functions on $X$ satisfies:

$$
\mathbb{C}(X) \cong K_{a(\tau)}
$$

Proof. Let $Y$ be the genus two surface with:

$$
\mathbb{C}(Y) \cong K_{a(\tau)}=\mathbb{C}(z, x) \text { with } z^{2}=\left(x^{2}-1\right)\left(x^{4}-a(\tau) x^{2}+1\right),
$$

and let $\psi$ be the $D_{8}$-action on $Y$ with $\psi(r)(z, x)=(z,-x)$ and $\psi(J)(z, x)=\left(i z / x^{3}, 1 / x\right)$. The quotient $Y / r$ has $\mathbb{C}(Y / r) \cong \mathbb{C}\left(z, x^{2}\right)$, a field isomorphic to $\mathbb{C}\left(E_{\tau}\right)$. The action $\psi(J)$ induces on the points of order two $E_{\tau}[2]^{*}$ is the transposition fixing $\left[w^{-1}(1)-\right.$ $\left.w^{-1}(\infty)\right]$. The isogeny $c_{\psi}$ is isomorphic to $i_{\tau}$ and Proposition 4.1 implies that $(Y, \psi) \sim$ $(X, \rho)$.

Remark. The field $K_{(-2 a+12) /(a+2)}$ is isomorphic to $K_{a}$. Equipping $K_{a}$ with the $D_{8^{-}}$ actions $\rho_{a}(z, x)=(z,-x)$ and $J(z, x)=\left(i z / x^{3}, 1 / x\right)$, the $D_{8}$-surfaces associated to $\left(K_{a}, \rho_{a}\right)$ and $\left(K_{(-2 a+12) /(a+2)}, \rho_{(-2 a+12) /(a+2)}\right)$ differ by the outer automorphism $\sigma$ of $D_{8}$. 
Cusps on $\mathcal{M}_{2}\left(D_{8}\right) / \sigma$. There are two cusps on $\mathcal{M}_{2}\left(D_{8}\right) / \sigma$, one of which maps to the cusp of the $(2,4, \infty)$-orbifold $\widetilde{\mathcal{M}}_{2}\left(D_{8}\right)^{p r} / \sigma$. For $(X, \rho) \in \mathcal{M}_{2}\left(D_{8}\right)$, let $\left|q_{\rho}\right|$ be the flat metric with singularities induced by a quadratic differential on $X / \rho(J)$ with simple poles at $X^{W} / \rho(J)$ and unit area $\int_{X / \rho(J)}\left|q_{\rho}\right|=1$.

Proposition 4.9. For a sequence of $D_{8}$-surfaces $\left(X_{i}, \rho_{i}\right) \in \mathcal{M}_{2}\left(D_{8}\right)$, the following are equivalent:

1. The $D_{8}$-surfaces $\left(X_{i}, \rho_{i}\right)$ diverge in $\widetilde{\mathcal{M}}_{2}\left(D_{8}\right)^{p r} / \sigma$.

2. The quotients $X_{i} / \rho_{i}(r)$ diverge in $\mathcal{M}_{1}$.

3. The surfaces $X_{i}$ tend to a stable limit with geometric genus zero.

4. The diameter of $\operatorname{Fix}_{W}\left(\rho_{i}(J)\right)$ in the metric space $\left(X_{i} / \rho_{i}(J),\left|q_{\rho_{i}}\right|\right)$ tends to zero.

Proof. Condition (1) and (2) are equivalent since the two maps $\widetilde{\mathcal{M}}_{2}\left(D_{8}\right)^{p r} \cong Y_{0}(2) \rightarrow$ $\mathcal{M}_{1}$ given by $(X, \rho) \mapsto X / r$ and $(X, \rho) \mapsto X / J r$ are proper. Now suppose (2) holds and $X_{i} / r$ diverge in $\mathcal{M}_{1}$. This happens if and only if there is a sequence of numbers $a_{i}$ tending to -2 so $\mathbb{C}\left(X_{i}\right) \cong K_{a_{i}}$. The stable limit $X_{\infty}$ has:

$$
\mathbb{C}\left(X_{\infty}\right) \cong K_{2}=\mathbb{C}(z, x) \text { with } z^{2}=\left(x^{2}-1\right)\left(x^{2}+1\right)^{2},
$$

and the geometric genus of $X_{\infty}$ is zero since $K_{2}=\mathbb{C}\left(\frac{z}{\left(x^{2}+1\right)(x-1)}\right)$. Conversely, a surface with geometric genus zero does not have compact Jacobian so (2) and (3) are equivalent.

Finally, the fields $\mathbb{C}\left(X_{i} / J\right) \subset K_{a_{i}}$ are generated by the function $g=x+1 / x$ and $g\left(\operatorname{Fix}_{W}(J)\right)=\{ \pm 2\}$. If $a_{i}$ tends to -2 , the remaining points in $X^{W}$ tend to 0 under $g$ and the diameter of $\operatorname{Fix}_{W}\left(\rho_{i}(J)\right)$ tends to zero in the metric space $\left(X_{i} / J,\left|q_{\rho_{i}}\right|\right)$. If $a_{i}$ tends to 2 , the remaining points in $X^{W}$ tend to \pm 2 under $g$ and the diameter of $\operatorname{Fix}_{W}\left(\rho_{i}(J)\right)$ tends to $\infty$ in $\left(X_{i} / J,\left|q_{\rho_{i}}\right|\right)$. This shows that (2) and (4) are equivalent. 

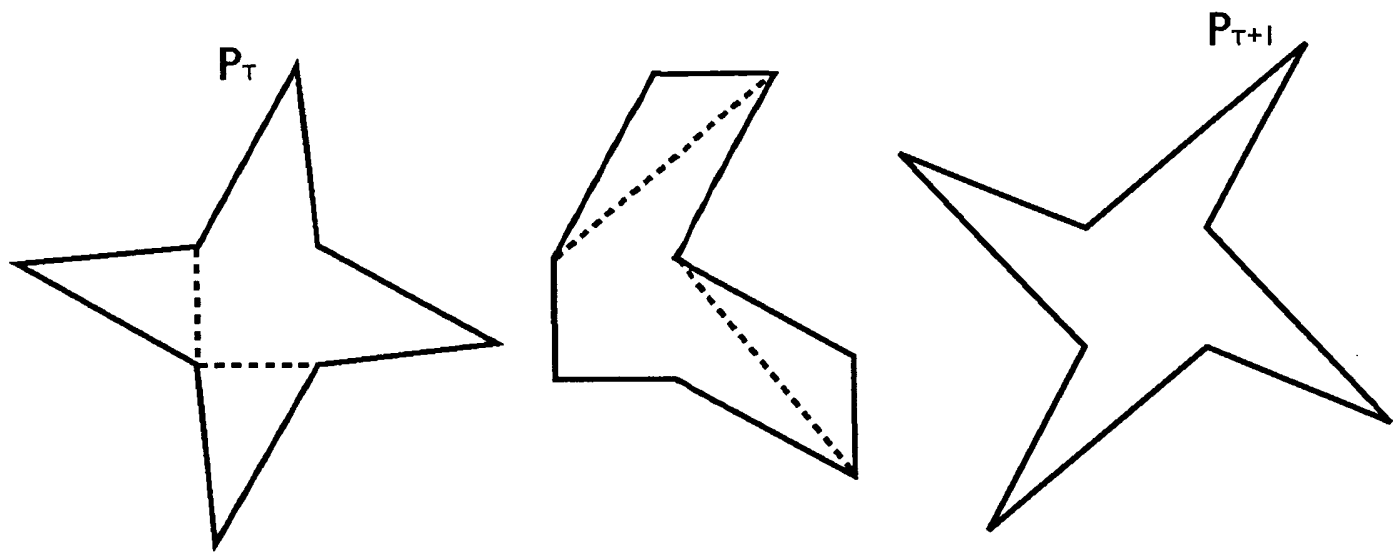

Figure 4-2: The surfaces $X_{\tau}$ and $X_{\tau+1}$ are isomorphic since the polygons $P_{\tau}$ and $P_{\tau+1}$ differ by a cut-and-paste operation.

Polygons. We now turn to the surfaces obtained from the polygons $P_{\tau}$ and $Q_{w}$. The following proposition, together with Propositions 4.7 and 4.8 completes the proof of Proposition 4.2:

Proposition 4.10. Fix $\tau \in U$. The surfaces $X_{\tau}$ and $Y_{w(\tau)}$ have proper $D_{8}$-actions $\rho_{X}$ and $\rho_{Y}$ with $c_{\rho_{X}}=c_{\rho_{Y}}=i_{\tau}$.

Proof. Let $J_{\tau}$ denote the order four automorphism on $X_{\tau}$ obtained from counterclockwise rotation the polygon $P_{\tau}$ and let $J_{w}$ denote the order four Deck transformation for the map on $Y_{w}$ depicted in Fig. 1-3. We will show that the maps $\tau \rightarrow\left(X_{\tau}, J_{\tau}\right)$ and $w \mapsto\left(Y_{w}, J_{w}\right)$ give maps:

$$
g_{U}: U / \sim_{U} \rightarrow \mathcal{M}_{2}\left(D_{8}\right) / \sigma \text { and } g_{V}: V / \sim_{V} \rightarrow \mathcal{M}_{2}\left(D_{8}\right) / \sigma
$$

with $\tau \sim_{U} \tau+1, \tau \sim_{U}-1 / 2 \tau, w \sim_{V} w+2$ and $w \sim_{V} w /(w+1)$. Since $U / \sim_{U}, V / \sim_{V}$ and $\mathcal{M}_{2}\left(D_{8}\right) / \sigma$ are all isomorphic to the $(2, \infty, \infty)$-orbifold, the maps $g_{U}$ and $g_{V}$ are isomorphisms. We will also show that $g_{U}(\tau)$ and $g_{V}(w)$ diverge in $\widetilde{\mathcal{M}}_{2}\left(D_{8}\right)^{p r} / \sigma$ as $\operatorname{Im}(\tau)$ and $\operatorname{Im}(w)$ tend to $\infty$, determining the isomorphisms $g_{U}$ and $g_{V}$ and showing that $X_{\tau}$ and $Y_{w(\tau)}$ have $D_{8}$-actions $\rho_{X}$ and $\rho_{Y}$ with $c_{\rho_{X}}=c_{\rho_{Y}}=i_{\tau}$.

By Proposition 3.3, the automorphisms $J_{\tau}$ and $J_{w}$ extend to actions of $D_{8}$ on $X_{\tau}$ and $Y_{w}$. By Proposition 4.5, the maps $\tau \mapsto\left(X_{\tau}, J_{\tau}\right)$ and $w \mapsto\left(Y_{w}, J_{w}\right)$ give 
holomorphic maps $g_{U}: U \rightarrow \mathcal{M}_{2}\left(D_{8}\right) / \sigma$ and $g_{V}: V \rightarrow M_{2}\left(D_{8}\right) / \sigma$.

The polygons $P_{\tau}$ and $P_{\tau+1}$ differ by a cut-and-paste operation (see Figure 4-2) and the polygons $P_{\tau}$ and $P_{-1 / 2 \tau}$ differ by Euclidean similarity. The surfaces $X_{\tau}, X_{\tau+1}$ and $X_{-1 / 2 \tau}$ are isomorphic with isomorphisms intertwining the actions of $J_{\tau}, J_{\tau+1}$ and $J_{-1 / 2 \tau}$ and the map $g_{U}$ descends to the quotient $U / \sim_{U}$. As $\operatorname{Im} \tau \rightarrow \infty$, the stable limit of $X_{\tau}$ has geometric genus zero and $g_{U}(\tau)$ diverges in $\mathcal{M}_{2}\left(D_{8}\right)^{p r} / \sigma$.

It is easy to check that the Veech group of $\left(Y_{i}, q_{i}\right)=\left(Q_{i}, d z^{2}\right) / \sim$ contains $\left(\begin{array}{ll}1 & 2 \\ 0 & 1\end{array}\right)$ and $\left(\begin{array}{ll}1 & 0 \\ 1 & 1\end{array}\right)$. The surfaces $Y_{w}, Y_{w+2}$ and $Y_{w /(w+1)}$ are all isomorphic with isomorphisms intertwining $J_{w}, J_{w+2}$ and $J_{w /(w+1)}$ and the map $g_{V}$ descends to the quotient $V / \sim_{V}$. The flat metric $\left|q_{\rho}\right|$ on $Y_{w} / J_{w}$ is induced by $\left|d z^{2}\right| / \operatorname{Im}(w)$ (Fig. 1-3) and the diameter of $\operatorname{Fix}_{W}\left(J_{w}\right)$ in $\left(Y_{w} / J_{w},\left|q_{\rho}\right|\right)$ is $1 / \sqrt{\operatorname{Im}(w)}$ which tends to zero as $\operatorname{Im}(w) \rightarrow \infty$. 


\section{Chapter 5}

\section{Endomorphisms}

Fix a smooth $D_{8}$-surface $(X, \rho)$ and let $E=\operatorname{Jac}(X / r)$. In this section we embed $\operatorname{End}(\operatorname{Jac}(X))$ as an order in $M_{2}(\operatorname{End}(E) \otimes \mathbb{Q})$, characterize the proper $D_{8}$-surfaces with complex multiplication and sort them by their orders for real multiplication to compute $e_{2}\left(W_{D}\right)$ and prove Theorem 1.1.

Theorem 1.8 describing the $D_{8}$-surfaces with complex multiplication follows from Proposition 4.2 and:

Proposition 5.1. The abelian variety $A_{\tau}$ has complex multiplication iff $\tau$ is imaginary quadratic.

Whenever $E$ has complex multiplication, $\rho(J)$ extends to complex multiplication by an order in $\operatorname{End}(E) \otimes \mathbb{Q}[i]$ giving an orbifold point on $\bigcup_{D} W_{D}$.

Proposition 5.2. Fix a $D_{8}$-surface $(X, \rho) \in \mathcal{M}_{2}\left(D_{8}\right)$ and suppose $\operatorname{Jac}(X)$ has complex multiplication. The action of $J$ on $\operatorname{Jac}(X)$ extends to complex multiplication $\iota$ by an order in $\mathbb{Q}(\sqrt{D}, i)$ and the pair $(X, \iota)$ gives an orbifold point on $\bigcup_{D} W_{D}$.

Propositions 3.4 and 5.2 complete our characterization of the orbifold points on $\bigcup_{D} W_{D}$ asserted in Corollary 1.6. The analogous results for $D_{12}$-surfaces show that the complex multiplication points of $\mathcal{M}_{2}\left(D_{12}\right)$ are labeled by orbifold points on $\bigcup_{D} X_{D}$ (see §A.1). Together with Proposition 3.2, this establishes our characterization the orbifold points on $\bigcup_{D} X_{D}$ in Theorem 1.5. 
Isogeny and endomorphism. The endomorphism rings of isogenous abelian varieties are related by the following proposition.

Proposition 5.3. Suppose $f: A \rightarrow B$ is an isogeny between principally polarized abelian varieties and $n$-times the polarization on $A$ is the polarization pulled back from $B$. The ring $\operatorname{End}(B)$ is isomorphic as an involutive algebra to the subring $R_{f} \subset \operatorname{End}(A) \otimes \mathbb{Q}$ given by:

$$
R_{f}=\frac{1}{n}\{\phi \in \operatorname{End}(A): \phi(\operatorname{ker}(n f)) \subset \operatorname{ker}(f)\}
$$

The ring $R_{f}$ satisfies $\frac{1}{n} \operatorname{End}(A) \supset R_{f} \supset n \operatorname{End}(A)$. Proposition 5.3 shows that $\operatorname{End}(A)$ is an order in $\operatorname{End}(B) \otimes \mathbb{Q}$ and that the rational endomorphism ring is an isogeny invariant. Propositions 5.1 and 5.2 follow:

Proof of Proposition 5.1. The abelian variety $A_{\tau}$ has a degree four isogeny to $E_{\tau} \times E_{\tau}$ and twice the polarization on $A_{\tau}$ is pulled back from the product polarization. When $\tau$ is imaginary quadratic, the ring $\operatorname{End}\left(A_{\tau}\right)$ is an order in $M_{2}(\mathbb{Q}(\tau))$ with $\left(\begin{array}{ll}a & b \\ c & d\end{array}\right)^{*}=\left(\begin{array}{l}\bar{a} \\ \bar{b} \\ \bar{d}\end{array}\right)$ and $A_{\tau}$ has complex multiplication by an order in $\mathbb{Q}\left(\tau, i=\left(\begin{array}{cc}0 & 1 \\ -1 & 0\end{array}\right)\right)$. When $\tau$ is not imaginary quadratic, $\operatorname{End}\left(A_{\tau}\right)$ is an order in $M_{2}(\mathbb{Q})$ with $\left(\begin{array}{ll}a & b \\ c & d\end{array}\right)^{*}=\left(\begin{array}{ll}a & c \\ b & d\end{array}\right)$ and $A_{\tau}$ does not have complex multiplication since every commutative subalgebra of $M_{2}(\mathbb{Q})$ has rank two over $\mathbb{Q}$.

Proof of Proposition 5.2. Suppose $(X, \rho)$ is a smooth $D_{8}$-surface and $\operatorname{Jac}(X)$ has complex multiplication. The center of $\operatorname{End}\left(A_{\tau}\right)$ is an order in $\mathbb{Q}(\tau)$ and the order four automorphism $\rho(J)$ extends to complex multiplication $\iota$ by an order $\mathcal{O}$ in $\mathbb{Q}(\tau, i)$. The $\mathcal{O}$-eigenforms have double zeros by Proposition 3.3, so the pair $(X, \iota)$ gives an orbifold point on $\bigcup_{D} W_{D}$.

Remark. The Jacobian $\operatorname{Jac}(X)$ for a $D_{12}$-surface $(X, \rho)$ is isogenous to $E \times E$ where $E=\operatorname{Jac}(X / r)(\S \mathrm{A} .1)$. The same argument shows that the $Z$-eigenforms on $D_{12^{-}}$ surfaces with complex multiplication are $\sigma$-eigenforms for orbifold points on $\bigcup_{D} X_{D}$, establishing our characterization of the orbifold points on Hilbert modular surfaces of Theorem 1.5. 
Proof of Proposition 5.3. We will show that the map:

$$
\begin{aligned}
\rho: \operatorname{End}(B) & \rightarrow \operatorname{End}(A) \otimes \mathbb{Q} \\
\phi & \mapsto \frac{1}{n} f^{*} \circ \phi \circ f
\end{aligned}
$$

is an injective homomorphism and has image $\rho(\operatorname{End}(B))=R_{f}$. The condition on the polarization implies that $f^{*} \circ f$ and $f \circ f^{*}$ are the multiplication by $n$ maps on $A$ and $B$ respectively and it is easy to check that $\rho$ is a homomorphism. The map $\rho$ is injective since rationally it in an isomorphism. The inverse for $\rho$ is given by $\rho^{-1}(\psi)=\frac{1}{n} f \circ \psi \circ f^{*}$.

The image of $\operatorname{End}(B)$ is contained in $R_{f}$ since:

$$
\operatorname{ker}(n f) \stackrel{f}{\rightarrow} B[n] \stackrel{\phi}{\rightarrow} B[n] \stackrel{f^{*}}{\rightarrow} \operatorname{ker}(f)
$$

for any $\phi \in \operatorname{End}(B)$. To see that the image of $\rho$ is all of $R_{f}$, fix $\psi \in \operatorname{End}(A) \otimes \mathbb{Q}$ satisfying $\psi(\operatorname{ker}(n f)) \subset \operatorname{ker}(f)$. The endomorphism $\phi=\frac{1}{n^{2}} f \circ \psi \circ f^{*}$ is integral on $B$ since:

$$
B\left[n^{2}\right] \stackrel{f^{*}}{\rightarrow} \operatorname{ker}(n f) \stackrel{\psi}{\rightarrow} \operatorname{ker}(f) \stackrel{f}{\rightarrow} 0 .
$$

Since $\rho(\phi)=\psi$, the image of $\rho$ is all of $R_{f}$.

The isomorphism between $\operatorname{End}(B)$ and $R_{f}$ has $\rho(\phi)^{*}=\rho\left(\phi^{*}\right)$ since $\left(f^{*} \phi f\right)^{*}=$ $f^{*} \phi^{*} f$.

Invertible modules over finite rings. The Jacobian of $X$ has a degree four isogeny $f: \operatorname{Jac}(X) \rightarrow E \times E$ with $f \circ f^{*}$ acting by multiplication by two on $E \times E$. In light of Proposition 5.3, if $E$ has complex multiplication by $\mathcal{O}_{C}$, the order for real multiplication on $X$ commuting with $J$ has discriminant $D=-C / 4,-C$ or $-4 C$. The actual order for real multiplication on $\operatorname{Jac}(X)$ commuting with $J$ depends only on $\operatorname{End}(E)$, the $M_{2}(\operatorname{End}(E))$-module $E[4] \times E[4]$ and the $\mathbb{Z}$-submodules $\operatorname{ker}(f)$ and $\operatorname{ker}(2 f)$.

To sort the complex multiplication points on $\mathcal{M}_{2}\left(D_{8}\right)$ by their orders for real 
multiplication we need to determine the possible $\mathcal{O}_{C}$-modules $E[4]$ :

Proposition 5.4. Let $\mathcal{O}$ be a quadratic order and I be a proper $\mathcal{O}$-ideal. The module $I / n I$ is isomorphic to $\mathcal{O} / n \mathcal{O}$ as $\mathcal{O}$-modules.

For imaginary quadratic orders, Proposition 5.4 can be deduced from the fact that $\mathbb{C} / I$ and $\mathbb{C} / J$ have algebraic models which are Galois conjugate whenever $I$ and $J$ and proper $\mathcal{O}$-ideals.

Proof. Since $\mathcal{O}$ is quadratic, the Galois conjugate $I^{\prime}$ is an inverse for $I$ and $I \otimes I^{\prime}$ is isomorphic to $\mathcal{O}$. The quotient $I / n I$ is also invertible and the invertible modules over $R=\mathcal{O} / n \mathcal{O}$ form a group $\operatorname{Pic}(R)$. We will show $\operatorname{Pic}(R)$ is trivial.

The ring $R$ is an Artin ring and by the structure theorem there are local Artin rings $R_{i}$ with:

$$
R=\prod_{i} R_{i} \text { and } \operatorname{Pic}(R)=\prod_{i} \operatorname{Pic}\left(R_{i}\right) .
$$

The group $\operatorname{Pic}\left(R_{i}\right)$ is trivial by Nakayama's lemma: if $I_{i}$ is an invertible $R_{i}$ module, and $m_{i}$ is the maximal ideal, $I_{i} / m_{i}$ is an invertible module over the field $R_{i} / m_{i}$. The $R_{i} / m_{i}$-vector space $I_{i} / m_{i} I_{i}$ is one dimensional and if $x \in I_{i}$ generates this vector space, $R_{i} x+m_{i} I_{i}=I_{i}$ which implies $I_{i}=R_{i} x$.

We are now ready to sort the complex multiplication points on $\mathcal{M}_{2}\left(D_{8}\right)$ by their orders for real multiplication.

Proof of Theorem 1.1. The only $D_{8}$-surface whose automorphism group is larger than $D_{8}$ is the surface obtained from the regular octagon labeling the point of order four on $W_{8}$ (cf. Proposition 3.1). For discriminants $D>8$, all of the orbifold points on $W_{D}$ are labeled by complex multiplication points on $\mathcal{M}_{2}\left(D_{8}\right)$ and have order two. To complete our proof, we need to determine the number of $D_{8}$-surfaces with real multiplication by $\mathcal{O}_{D}$ commuting with $\rho(J)$.

For each $C \equiv 0$ or $1 \bmod 4$, set:

$$
\mathcal{O}_{C}=\frac{\mathbb{Z}[x]}{x^{2}-C x+\frac{C(C-1)}{4}}
$$




\begin{tabular}{|c|c|c|c|}
\hline$C \bmod 16$ & $D_{1}$ & $D_{2}$ & $D_{3}$ \\
\hline 0 & $-4 C$ & $-C$ & $-4 C$ \\
4 & $-4 C$ & $-4 C$ & $-C$ \\
8 & $-4 C$ & $-C$ & $-4 C$ \\
12 & $-4 C$ & $-4 C$ & $-C / 4$ \\
1 or 9 & $-4 C$ & $-4 C$ & $-4 C$ \\
5 or 13 & $-4 C$ & $-4 C$ & $-4 C$ \\
\hline
\end{tabular}

Table 5.1: The elliptic curve $E=\mathbb{C} / \mathcal{O}_{C}$ is covered by three $D_{8}$-surfaces $\left(X_{i}, \rho_{i}\right)$. The discriminant $D_{i}$ of the order for real multiplication on $\operatorname{Jac}\left(X_{i}\right)$ commuting with $J$ is computed using Proposition 5.3.

The ring $\mathcal{O}_{C} / 4 \mathcal{O}_{C}$ depends only on $C \bmod 16$. Let $P_{1}=1, P_{2}=x$ and $P_{3}=x+1$ be the points of order two in $\mathcal{O}_{C} / 2 \mathcal{O}_{C}$ and let $\left(X_{i}, \rho_{i}\right)$ be the $D_{8}$-surface in $\widetilde{\mathcal{M}}_{2}\left(D_{8}\right)^{p r}$ with $X_{i} / \rho_{i}(r) \cong \mathbb{C} / \mathcal{O}_{C}$ and $\operatorname{ker}\left(c_{\rho_{i}}\right)$ generated by $P_{i}$. In Table 5.1 we compute the discriminant $D_{i}$ of the order for real multiplication on $\operatorname{Jac}\left(X_{i}\right)$ commuting with $J$ using Proposition 5.3. Most of the entries in Table 5.1 are determined by the observation that $\operatorname{Jac}\left(X_{i}\right)$ has complex multiplication by an order containing $\mathcal{O}_{C}[i]$ iff the $\mathcal{O}_{C}$-module $P_{i} \mathcal{O}_{C} / 2 \mathcal{O}_{C}$ is a proper submodule of $\mathcal{O}_{C} / 2 \mathcal{O}_{C}$.

For any genus one surface $E$ with complex multiplication by $\mathcal{O}_{C}$, we have $E[4] \cong$ $\mathcal{O}_{C} / 4$ by Proposition 5.4. With an appropriate ordering, the three $D_{8}$-surfaces covering $E$ have the same orders for real multiplication as the three $D_{8}$-surfaces covering $\mathbb{C} / \mathcal{O}_{C}$ by Proposition 5.3. The formula for $e_{2}\left(W_{D}\right)$ in Table 1.1 follows easily. Note that the factor of two in the formula for $e_{2}\left(W_{D}\right)$ comes from the fact that the $D_{8^{-}}$ surfaces $(X, \rho)$ and $(X, \rho \circ \sigma)$ label the same orbifold point on $\bigcup_{D} W_{D}$. 


\section{Chapter 6}

\section{Spin}

For a number $\tau \in U$, let $\omega_{\tau} \in \Omega\left(X_{\tau}\right)$ be the holomorphic one-form with double zero on the surface $X_{\tau}$ obtained by gluing together opposite sides on $P_{\tau}$ :

$$
\left(X_{\tau}, \omega_{\tau}\right)=\left(P_{\tau}, d z\right) / \sim
$$

In this section we compute the spin structure $q: H_{1}\left(X_{\tau}, \mathbb{Z} / 2 \mathbb{Z}\right) \rightarrow \mathbb{Z} / 2 \mathbb{Z}$ coming from the form $\omega_{\tau}$ and then, for $D \equiv 1 \bmod 8$, sort the orbifold points on $W_{D}$ by their spin component.

Spin structure. Let $a$ be the number $\frac{\tau-1+i}{2}$ and $x_{t}$ be the homology class in $H_{1}\left(X_{\tau}, \mathbb{Z}\right)$ with period $t \in \mathbb{Z}[i] \oplus a \mathbb{Z}[i]$ under the one-form $\omega_{\tau}$.

Proposition 6.1. The spin structure $q$ on $X_{\tau}$ obtained from the form with double zero $\omega_{\tau}$ has:

$$
q\left(k_{1} x_{1}+k_{2} x_{i}+k_{3} x_{a}+k_{4} x_{a i}\right)=k_{1}^{2}+k_{2}^{2}+k_{1} k_{3}+k_{2} k_{4}+k_{3} k_{4} .
$$

Proof. Isotoping representatives away from the zero $Z\left(\omega_{\tau}\right)$ and computing the degree of the Gauss map shows that the spin structure $q$ associated to $\omega_{\tau}$ satisfies:

$$
q\left(x_{1}\right)=q\left(x_{i}\right)=1 \text { and } q\left(x_{a}\right)=q\left(x_{a i}\right)=0 .
$$


From the relation $q(x+y)=q(x)+q(y)+\langle x, y\rangle$, any basis $x_{n}$ of $H_{1}\left(X_{\tau}, \mathbb{Z} / 2 \mathbb{Z}\right)$ has:

$$
q\left(\sum_{n} k_{n} x_{n}\right)=\sum_{n} k_{n}^{2} q\left(x_{n}\right)+\sum_{l<n} k_{l} k_{n}\left\langle x_{l}, x_{n}\right\rangle .
$$

Computing the intersection pairing on the basis $\left\{x_{1}, x_{i}, x_{a}, x_{a i}\right\}$ gives the stated formula for $q$.

Spin homomorphism. Now fix an integer $D \geq 9$ with $D \equiv 1 \bmod 8$ and let $f$ be the conductor of $D$. An ideal $I \subset \mathcal{O}_{-4 D}$ has norm $\mathrm{Nm}(I)=2^{k} l$ with $l$ odd. We define:

$$
\epsilon_{0}(I)=\frac{l-1}{2} \bmod 2 .
$$

Proposition 6.2. The number $\epsilon_{0}(I)$ depends only on the ideal class of $I$ and defines a spin homomorphism:

$$
\epsilon_{0}: H(-4 D) \rightarrow \mathbb{Z} / 2 \mathbb{Z}
$$

The spin homomorphism $\epsilon_{0}$ is the zero map iff $D$ is a square.

Proof. Any $x \in \mathcal{O}_{-4 D}$ has norm $\operatorname{Nm}(x)=x_{1}^{2}+x_{2}^{2} D=2^{k} l$ with $l \equiv 1 \bmod 4$. If the ideals $I$ and $J$ are in the same ideal class, they satisfy $x I=y J$ for some $x$ and $y$ in $\mathcal{O}_{-4 D}$ and $\epsilon_{0}(I)=\epsilon_{0}(J)$. The map $\epsilon_{0}$ is a homomorphism since the norm of ideals is a homomorphism.

Now suppose $D=f^{2}$ is a square. Any $\mathcal{O}_{-4 D}$-ideal class has a representative of the form $I=x \mathbb{Z} \oplus(f i-y) \mathbb{Z}$. Since $I$ is an ideal, $x$ divides $f^{2}+y^{2}$ and since $I$ is proper $\operatorname{gcd}\left(x, y,\left(f^{2}+y^{2}\right) / x\right)=1$. If an odd prime $p$ divides $\mathrm{Nm}(I)$, then $p$ divides $x^{2}, f^{2}+y^{2}$, and $y$. Since $f^{2} \equiv-y^{2} \bmod p$ and $p$ does not divide both $f$ and $y,-1$ is a square $\bmod p, p \equiv 1 \bmod 4$ and $\epsilon_{0}(I)=0$.

If $D$ is not a square, $D=p_{1}^{k_{1}} p_{2}^{k_{2}} \ldots p_{n}^{k_{n}}$ with $p_{l}$ distinct odd primes and $k_{1}$ odd. By Dirichlet's theorem, there is a prime $p$ with:

- $p \equiv 3 \bmod 4$,

- $p \equiv 1 \bmod p_{l}$ for $l>1$, and 
- $\left(\frac{p}{p_{1}}\right)=-1$

Quadratic reciprocity gives $\left(\frac{-D}{p}\right)=1$. If $x^{2} \equiv-D \bmod p$, then $I=p \mathbb{Z} \oplus(\sqrt{-D}-x) \mathbb{Z}$ is an $\mathcal{O}_{-4 D^{-i d e a l}}$ and has norm $p$ and $\epsilon_{0}(I)=1$.

Remark. When $D \equiv 1 \bmod 8$, the ideal (2) ramifies in $\mathcal{O}_{-4 D}$ and there is a prime ideal $P$ with $P^{2}=(2)$. Since $\operatorname{Nm}(P)=2$, we have $\epsilon_{0}(P)=0$. The ideal classes represented by $I=\mathbb{Z} \oplus \tau \mathbb{Z}$ and $J=\mathbb{Z} \oplus-1 / 2 \tau \mathbb{Z}$ satisfy $[I]=[P J]$. This is related to the fact the polygonal stars $P_{\tau}$ and $P_{-1 / 2 \tau}$ give the same point on $W_{D}$ and so must have the same spin invariant.

Spin and orbifold points. As before, fix a discriminant $D \geq 9$ with $D \equiv 1 \bmod 8$ and conductor $f$.

Proposition 6.3. Suppose $\left(X_{\tau}, \omega_{\tau}\right)$ is an eigenform for real multiplication $\iota_{\tau}$ by $\mathcal{O}_{D}$ obtained from the polygon $P_{\tau}$ and let $I=\mathbb{Z} \oplus \tau \mathbb{Z}$. The spin invariant for $\left(X_{\tau}, \iota_{\tau}\right)$ is given by:

$$
\epsilon\left(X_{\tau}, \iota_{\tau}\right)=\frac{f+1}{2}+\epsilon_{0}(I) \bmod 2 .
$$

The formula for $e_{2}\left(W_{D}^{\epsilon}\right)$ stated in Theorem 1.2 follows from Propositions 6.2 and 6.3 .

Proof. According to Table 5.1, $\operatorname{Jac}\left(X_{\tau}\right)$ has real multiplication by $\mathcal{O}_{D}$ with eigenform $\omega_{\tau}$ iff $I$ is a proper $\mathcal{O}_{-4 D}$-ideal and $1+\sqrt{-D} \in 2 I$.

Since $I$ is an ideal, $\sqrt{-D}=x \tau+y$ for some $x$ and $y \in \mathbb{Z}, x$ divides $D+y^{2}$ and $I$ has the same class as $I_{0}=x \mathbb{Z} \oplus(\sqrt{-D}-y) \mathbb{Z}$. Since $I$ is proper, $\operatorname{gcd}\left(x, y,\left(D+y^{2}\right) / x\right)=1$ and the norm of $I_{0}$ is $x$ up to a factor of two. The condition $1+\sqrt{-D} \in 2 I$ ensures $x \equiv 2 \bmod 4$ and $\epsilon_{0}(I) \equiv \frac{x-2}{4} \bmod 2$.

To compute the spin invariant $\epsilon\left(X_{\tau}, \omega_{\tau}\right)$, we need to determine the subspace $V=$ $\operatorname{Im}\left(\frac{f+\sqrt{D}}{2}\right)$ of $H_{1}(X, \mathbb{Z} / 2 \mathbb{Z})$ and evaluate $\operatorname{Arf}\left(\left.q\right|_{V}\right)$. The subspace $V$ is spanned by $v$ and $i v$ where:

$$
v=\frac{f-i \sqrt{-D}}{2} \equiv \frac{2 f+x}{4}+i \frac{x-2 y}{4}+a i \bmod 2
$$


Since $q(v)=q(i v)$, we have by Proposition 6.1:

$$
\epsilon\left(X_{\tau}, \omega_{\tau}\right)=q(v)^{2}=\frac{f+1}{2}+\epsilon_{0}(I) \bmod 2 .
$$

Remark. For square discriminants $D=f^{2}$, there is an elementary argument that shows $e_{2}\left(W_{D}^{(f-1) / 2}\right)=0$. When the surface $X_{\tau}$ is labeled by an orbifold point on $W_{D}$, the number $\tau$ is in $\mathbb{Q}(i)$ and rescaling $P_{\tau}$, we can exhibit $X_{\tau}$ as the quotient of a polygon with vertices in $\mathbb{Z}[i]$ and area $f$. The area determines the number of points in $X^{W}$ lying on the lattice $\mathbb{Z}[i]$ which in turn determines the spin invariant. 


\section{Chapter 7}

\section{Genus}

Together with [Ba, Mc3], Theorems 1.1 and 1.2 complete the determination of the homeomorphism type of $W_{D}$. For non-square $D$, we will show that the homeomorphism type of $W_{D}$ can be determined from the homeomorphism type of the Hilbert modular surface $X_{D}$ and the product locus $P_{D}$ :

Proposition 7.1. For non-square $D>8$, the homeomorphism type of $W_{D}$ is determined by the orbifold Euler characteristic $\chi\left(X_{D}\right)$, the number of cusps $C\left(P_{D}\right)$ on $P_{D}$, the number of orbifold points of order two $e_{2}\left(X_{D}\right)$ and $e_{2}\left(P_{D}\right)$ and $D \bmod 8$.

We will use our formula to give bounds for the genus of $W_{D}$ and its components.

Proposition 7.2. For any $\epsilon>0$, there are positive constants $C_{\epsilon}$ and $N_{\epsilon}$ so:

$$
C_{\epsilon} D^{3 / 2+\epsilon}>g(V)
$$

whenever $V$ is a component of $W_{D}$ and $D>N_{\epsilon}$.

We will also give effective lower bounds.

Proposition 7.3. Suppose $D>0$ is a discriminant and $V$ is a component of $W_{D}$. If $D$ is not a square, the genus of $V$ satisfies:

$$
g(V)>D^{3 / 2} / 600-D / 6-D^{3 / 4} / 2-75 .
$$


If $D$ is a square, the genus of $V$ satisfies:

$$
g(V)>D^{3 / 2} / 240-D-D^{3 / 4} / 2-75 .
$$

The components of $\bigcup_{D} W_{D}$ with genus $g \leq 4$ are all listed in $\S$ A.2.

Corollary 7.4. The components of $\bigcup_{D} W_{D}$ with genus $g \leq 4$ all lie on $\bigcup_{D \leq 121} W_{D}$.

Proof. The bounds in Proposition 7.3 show that $g(V)>4$ whenever $D>17500$ for non-square $D$ and $D>250^{2}$ for square $D$. The remaining discriminants were checked by computer.

Euler characteristic of $X_{D}$ and $W_{D}$. The Hilbert modular surface $X_{D}$ has a meromorphic modular form with a simple zero along $W_{D}$ and simple pole along $P_{D}$. This gives a simple relationship between the orbifold Euler characteristics of $W_{D}, P_{D}$ and $X_{D}$ and a modular curve $S_{D}$ in the boundary of $X_{D}$. The curve $S_{D}$ is empty unless $D=f^{2}$ is a square, in which case $S_{D} \cong X_{1}(f)$.

Theorem 7.5 ([Ba] Cor. 10.4). The Euler characteristic of $W_{D}$ satisfies:

$$
\chi\left(W_{D}\right)=\chi\left(P_{D}\right)-2 \chi\left(X_{D}\right)-\chi\left(S_{D}\right) .
$$

For a discriminant $D$, define:

$$
F(D)=\prod_{p \mid f}\left(1-\left(\frac{D_{0}}{p}\right) p^{-2}\right) .
$$

where $f$ is the conductor of $\mathcal{O}_{D}, D_{0}=D / f^{2}$ is the discriminant of the maximal order in $\mathbb{Q}(\sqrt{D})$ and the product is over primes dividing $f$. The number $F(D)$ satisfies $1>F(D)>\zeta_{\mathbb{Q}}(2)^{-1}>6 / 10$.

For square discriminants, $\chi\left(S_{D}\right)=-f^{2} F(D) / 12$ and the Euler characteristic of 
$W_{D}$ and its components are given by ([Ba] Thm. 1.4):

$$
\begin{aligned}
& \chi\left(W_{f^{2}}\right)=-f^{2}(f-1) F(D) / 16, \\
& \chi\left(W_{f^{2}}^{0}\right)=-f^{2}(f-1) F(D) / 32, \text { and } \\
& \chi\left(W_{f^{2}}^{1}\right)=-f^{2}(f-3) F(D) / 32 .
\end{aligned}
$$

For non-square discriminants, $\chi\left(S_{D}\right)=0$ and $\chi\left(P_{D}\right)=-\frac{5}{2} \chi\left(X_{D}\right)$ giving $\chi\left(W_{D}\right)=$ $-\frac{9}{2} \chi\left(X_{D}\right)$. The Euler characteristic $\chi\left(X_{D}\right)$ can be computed from ([Ba] Thm. 2.12):

$$
\chi\left(X_{D}\right)=2 f^{3} \zeta_{D_{0}}(-1) F(D)
$$

Here $\zeta_{D_{0}}$ is the Dedekind-zeta function and can be computed from Siegel's formula ([Bru], Cor. 1.39):

$$
\zeta_{D_{0}}(-1)=\frac{1}{60} \sum_{e^{2}<D_{0}, e \equiv D_{0} \bmod 2} \sigma\left(\frac{D_{0}-e^{2}}{4}\right)
$$

where $\sigma(n)$ is the sum of the divisors of $n$. For reducible $W_{D}$, the spin components satisfy $\chi\left(W_{D}^{0}\right)=\chi\left(W_{D}^{1}\right)=\frac{1}{2} \chi\left(W_{D}\right)([\mathrm{Ba}] \mathrm{Thm} .1 .3)$.

The well-known bound $\sigma(n)=o\left(n^{1+\epsilon}\right)$ gives constants $C_{\epsilon}$ and $N_{\epsilon}$ so:

$$
C_{\epsilon} D^{3 / 2+\epsilon}>\chi\left(X_{D}\right)
$$

whenever $D>N_{\epsilon}$. Using $\sigma(n)>n+1$ and $F(D)>6 / 10$ gives:

$$
\chi\left(X_{D}\right)>D^{3 / 2} / 300
$$

We can now prove the upper bounds for the genus of $W_{D}$ :

Proof of Proposition 7.2. For square discriminants $D=f^{2}$, we have $\left|\chi\left(W_{D}\right)\right|<f^{3}$. For non-square discriminants, the bounds for $\chi\left(X_{D}\right)$ and the formula $\chi\left(W_{D}\right)=$ $-9 \chi\left(X_{D}\right) / 2$ gives $\left|\chi\left(W_{D}\right)\right|=O\left(D^{3 / 2+\epsilon}\right)$. Since $W_{D}$ has one or two components, 
$g\left(W_{D}\right)=O\left(\left|\chi\left(W_{D}\right)\right|\right)$

The modular curve $P_{D}$. The modular curve $P_{D}$ is isomorphic to:

$$
\left(\bigsqcup_{(e, l, m)} Y_{0}(m)\right) / g
$$

where the union is over triples of integers $(e, l, m)$ with:

$$
D=e^{2}+4 l^{2} m, \quad l, m>0, \quad \text { and } \operatorname{gcd}(e, l)=1,
$$

and $g$ is the automorphism sending the degree $m$ isogeny $i$ on the component labeled by $(e, l, m)$ to the isogeny $i^{*}$ on the $(-e, l, m)$-component (cf. [Mc3] Thm. 2.1). The isogeny $i: E \rightarrow F$ on the $(e, l, m)$-component corresponds to the abelian variety $B=E \times F$ with $\mathcal{O}_{D}$ generated by $\iota\left(\frac{e+\sqrt{D}}{2}\right)=\left(\begin{array}{cc}e & i^{*} \\ i & 0\end{array}\right)$.

The components of $P_{D}$ are labeled by triples $(e, l, m)$ as above subject to the additional condition $e \geq 0$. We will need the following bound on the number of such triples:

Proposition 7.6. The number of components of $P_{D}$ satisfies:

$$
h_{0}\left(P_{D}\right) \leq D^{3 / 4}+150
$$

Proof. Let $l(n)$ denote the largest integer whose square divides $n$ and let $f(n)=$ $d(l(n))$ be the number of divisors of $l(n)$. The function $f$ is multiplicative and the number of triples $(e, l, m)$ with $e$ fixed is bounded above by $f\left(\frac{D-e^{2}}{4}\right)$. There is a finite set $S$ of natural numbers $n$ for which $f(n)>n^{1 / 4}$ (they are all divisors of $2^{12} 3^{6} 5^{4} 7^{2} 11^{2}$ ) since $d(n)$ is $o\left(n^{\epsilon}\right)$ for any $\epsilon>0$ and it is easy to check that $\sum_{n \in S} f(n)-n^{1 / 4}<150$. The asserted bound on $h^{0}\left(P_{D}\right)$ follows from:

$$
h_{0}\left(P_{D}\right) \leq \sum_{\substack{e \equiv D \bmod 2 \\ 0 \leq e<\sqrt{D}}} f\left(\frac{D-e^{2}}{4}\right) \leq 150+\sum_{e}\left(\frac{D-e^{2}}{4}\right)^{1 / 4} .
$$


Cusps on $W_{D}$ and $P_{D}$. Let $C_{1}\left(W_{D}\right)$ and $C_{2}\left(W_{D}\right)$ be the number of one- and twocylinder cusps on $W_{D}$ respectively and $C\left(W_{D}\right)=C_{1}\left(W_{D}\right)+C_{2}\left(W_{D}\right)$ be the total number of cusps. The cusps on $W_{D}$ were first enumerated and sorted by component in [Mc3]:

Proposition 7.7. For non-square discriminants, the number of cusps on $W_{D}$ is equal to the number of cusps on $P_{D}$ :

$$
C\left(W_{D}\right)=C_{2}\left(W_{D}\right)=C\left(P_{D}\right)
$$

and $C\left(W_{D}^{0}\right)=C\left(W_{D}^{1}\right)$ when $W_{D}$ is reducible. For square discriminants $D=f^{2}$, the number of one- and two-cylinder cusps satisfy:

$$
C_{2}\left(W_{f^{2}}\right)<C\left(P_{f^{2}}\right) \text { and } C_{1}\left(W_{f^{2}}\right)<f^{2} / 3 \text {. }
$$

When $f$ is odd, $\left|C\left(W_{f^{2}}^{1}\right)-C\left(W_{f^{2}}^{0}\right)\right|<7 f^{2} / 12$.

Proof. Except for the explicit bounds on $C_{1}\left(W_{f^{2}}\right)$ and $\left|C\left(W_{f^{2}}^{1}\right)-C\left(W_{f^{2}}^{0}\right)\right|$, the claims in the proposition follow from the enumeration of cusps on $P_{D}$ and $W_{D}$ in $[\mathrm{Ba}]$, Section 3.1. We will sketch another argument for the relationship between $C\left(W_{D}\right)$ and $C\left(P_{D}\right)$. The cusps on $W_{D}$ are labeled by splitting saddle connections for eigenforms ([Mc3], §3). For a point $(X, \iota) \in W_{D}$ near a cusp, there is a unique short splitting saddle connection and the splitting gives a point on $P_{D}$ near a cusp. This gives a correspondence between the two-cylinder cusps on $W_{D}$ and the cusps on $P_{D}$. One can show that this correspondence is always injective and is bijective whenever $D$ is not a square by showing there is an inverse correspondence defined on a subset of the cusps of $P_{D}$.

We now turn to the bounds on $C_{1}\left(W_{f^{2}}\right)$ and $\left|C\left(W_{f^{2}}^{1}\right)-C\left(W_{f^{2}}^{0}\right)\right|$. When $D$ is not a square, there are no one-cylinder cusps and when $D=f^{2}$ is a square, the one-cylinder cusps are parametrized by cyclically ordered triples $(a, b, c)$ with (cf. [Mc3] Theorem 
A.1):

$$
f=a+b+c, a, b, c>0 \text { and } \operatorname{gcd}(a, b, c)=1 .
$$

Reordering $(a, b, c)$ so $a<b$ and $a<c$ ensures that $a<f / 3$ and $b<f$, giving $C_{1}\left(W_{D}\right)<f^{2} / 3=D / 3$. The difference in the number of two cylinder cusps is given by (Theorem A.4 in [Mc3]):

$$
C_{2}\left(W_{D}^{0}\right)-C_{2}\left(W_{D}^{1}\right)=\sum_{b+c=f, 0<c<b} \phi(\operatorname{gcd}(b, c)),
$$

which is smaller than $D / 4$ using $c<f / 2$ and $\phi(\operatorname{gcd}(b, c))<f / 2$. The bound asserted for $\left|C\left(W_{D}^{0}\right)-C\left(W_{D}^{1}\right)\right|$ follows.

Orbifold points on $W_{D}$. Our formula for $e_{2}\left(W_{D}\right)$ gives:

Theorem 7.8. The number of points of order two on $W_{D}$ satisfies:

$$
e_{2}\left(W_{D}\right)=e_{2}\left(X_{D}\right)-e_{2}\left(P_{D}\right) \text { and } e_{2}\left(W_{D}\right)<\frac{4}{3} D
$$

For non-square $D$, Theorem 7.8 establishes Proposition 7.1 together with Proposition 7.7 and the enumeration of the irreducible components of $W_{D}$ in [Mc3].

Proof. Theorem 1.5 and Corollary 1.6 say that every orbifold point of order two on $X_{D}$ is an orbifold point on $P_{D}$ or $W_{D}$, giving $e_{2}\left(W_{D}\right)=e_{2}\left(X_{D}\right)-e_{2}\left(P_{D}\right)$. The bound for $e_{2}\left(W_{D}\right)$ follows from the bound $h(-D)<2 D / 3$ (§2) for discriminants $-D<0$ and the formula in Theorem 1.1.

Lower bounds, non-square discriminants. Now suppose $D$ is a non-square discriminant and $D>8$ so all of the orbifold points on $W_{D}$ have order two. Using the formula for $\chi\left(W_{D}\right)$, the equality $C\left(P_{D}\right)=C\left(W_{D}\right)$, and $\chi\left(S_{D}\right)=0$ and ignoring several terms which contribute positively to the $g\left(W_{D}\right)$ gives:

$$
g\left(W_{D}\right)>\chi\left(X_{D}\right)-h^{0}\left(P_{D}\right)-e_{2}\left(W_{D}\right) / 4 .
$$


Combining the bound above with $\chi\left(X_{D}\right)>D^{3 / 2} / 300, h_{0}\left(P_{D}\right)<D^{3 / 4}+150, e_{2}\left(W_{D}\right)<$ $\frac{4}{3} D$ and $g(V) \geq \frac{1}{2} g\left(W_{D}\right)$ whenever $V$ is a component of $W_{D}$ gives the bound in Proposition 7.3.

Lower bounds, square discriminants. Now suppose $D=f^{2}$. Using the formula for $\chi\left(W_{D}\right)$ in terms of $\chi\left(X_{D}\right), \chi\left(P_{D}\right)$ and $\chi\left(S_{D}\right)$, the bound $C_{2}\left(W_{D}\right)<C\left(P_{D}\right)$ and ignoring some terms which contribute positively to $g\left(W_{D}\right)$ gives:

$$
g\left(W_{D}\right) \geq \chi\left(X_{D}\right)-h_{0}\left(P_{D}\right)-e_{2}\left(W_{D}\right) / 4+\chi\left(S_{D}\right) / 2-C_{1}\left(W_{D}\right) / 2
$$

As before we have $h_{0}\left(P_{D}\right)<D^{3 / 4}+150, e_{2}\left(W_{D}\right)<\frac{4}{3} D$ and $C_{1}\left(W_{D}\right)<D / 3$. By Theorem 2.12 and Proposition 10.5 of $[\mathrm{Ba}]$ and using $\zeta_{\mathbb{Q}}(2)>6 / 10$, we have $\chi\left(X_{D}\right)+$ $\chi\left(S_{D}\right) / 2>D^{3 / 2} / 120-D / 40$ so long as $D>36$, giving:

$$
g\left(W_{D}\right) \geq D^{3 / 2} / 120-3 D / 5-D^{3 / 4}-150 .
$$

Finally, to bound $g(V)$ when $V$ is a component of $W_{D}$, we bound the difference:

$$
\left|g\left(W_{D}^{0}\right)-g\left(W_{D}^{1}\right)\right| \leq\left|\frac{\chi\left(W_{D}^{1}\right)-\chi\left(W_{D}^{0}\right)}{2}\right|+\left|\frac{C\left(W_{D}^{1}\right)-c\left(W_{D}^{0}\right)}{2}\right|+e_{2}\left(W_{D}\right) / 4
$$

We have seen that $\left|C\left(W_{D}^{1}\right)-C\left(W_{D}^{0}\right)\right|<7 D / 12$ and $e_{2}\left(W_{D}\right) / 4<D / 3$. Theorem 1.4 of $[\mathrm{Ba}]$ gives $\left|\chi\left(W_{D}^{0}\right)-\chi\left(W_{D}^{1}\right)\right|<D / 16$ and $\left|g\left(W_{D}^{1}\right)-g\left(W_{D}^{0}\right)\right|<7 D / 10$. The bound asserted for $g(V)$ in Proposition 7.3 follows. 


\section{Appendix A}

\section{The $D_{12}$-family}

\section{A.1 The $D_{12}$-family}

In this section we will describe the surfaces in $\mathcal{M}_{2}\left(D_{12}\right)$. For a smooth surface $X \in$ $\mathcal{M}_{2}$, the following are equivalent:

- Automorphisms. The automorphism group $\operatorname{Aut}(X)$ admits an injective homomorphism $\rho: D_{12} \rightarrow \operatorname{Aut}(X)$.

- Algebraic curves. The field of functions $\mathbb{C}(X)$ is isomorphic to the field:

$$
\widetilde{K}_{a}=\mathbb{C}(z, x) \text { with } z^{2}=x^{6}-a x^{3}+1
$$

for some $a \in \mathbb{C} \backslash\{ \pm 2\}$.

- Jacobians. The Jacobian $\operatorname{Jac}(X)$ is isomorphic to the principally polarized abelian variety:

$$
\widetilde{A}_{\tau}=\mathbb{C}^{2} / \widetilde{\Lambda}_{\tau}
$$

where $\widetilde{\Lambda}_{\tau}=\mathbb{Z}\left\langle\left(\begin{array}{c}1 \\ 1 / \sqrt{3}\end{array}\right),\left(\begin{array}{c}\tau \\ \sqrt{3} \tau\end{array}\right),\left(\begin{array}{c}1 \\ -1 / \sqrt{3}\end{array}\right),\left(\begin{array}{c}\tau \\ -\sqrt{3} \tau\end{array}\right)\right\rangle$ and is polarized by the symplectic form $\left\langle\left(\begin{array}{l}a \\ b\end{array}\right),\left(\begin{array}{l}c \\ d\end{array}\right)\right\rangle=\frac{\operatorname{Im}(a \bar{c}+b \bar{d})}{2 \operatorname{Im} \tau}$.

- Hexagonal pinwheels. The surface $X$ is isomorphic to the surface $\widetilde{X}_{\tau}$ obtained by gluing the hexagonal pinwheel $H_{\tau}$ (Fig. A-1) to $-H_{\tau}$ for some $\tau$ in the 


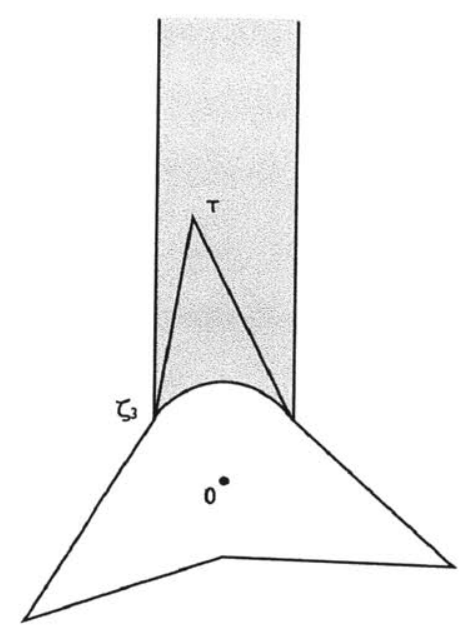

Figure A-1: For $\tau$ in the shaded domain $\widetilde{U}$, the hexagonal pinwheel $H_{\tau}$ has vertices lying on two equilateral triangles. Gluing together sides on $H_{\tau}$ and $-H_{\tau}$ by translation gives a genus two surface admitting an action of $D_{12}$. The one form induced by $d z$ is a $Z$-eigenform.

domain:

$$
\widetilde{U}=\left\{\tau \in \mathbb{H}: \tau \neq \zeta_{12} \text { or } \zeta_{12}^{5},|\operatorname{Re} \tau| \leq \frac{1}{2} \text { and }|\tau| \geq 1\right\}
$$

- Parallelograms. The surface $X$ is isomorphic the degree six cyclic cover $\widetilde{Y}_{w}$ of the double of a parallelogram with vertices $\{0,1, w / 2,(w+1) / 2\}$ depicted in Fig. A-2 for some $w$ in the domain:

$$
\widetilde{V}=\left\{w \in \mathbb{H}:|\operatorname{Re} \tau| \leq \frac{1}{2},|\tau| \geq 1 \text { and }|\tau-1| \geq 1\right\}
$$

It is straightforward to identify the action of $D_{12}$ on the surfaces described above. The field $\widetilde{K}_{a}$ has automorphisms $Z(z, x)=\left(-z, \zeta_{3} x\right)$ and $r(z, x)=\left(z / x^{3}, 1 / x\right)$. The polarized lattice $\widetilde{\Lambda}_{\tau}$ is preserved by the linear transformations $r=\left(\begin{array}{cc}1 & 0 \\ 0 & -1\end{array}\right)$ and $Z=$ $\frac{1}{2}\left(\begin{array}{cc}1 & -\sqrt{3} \\ \sqrt{3} & 1\end{array}\right)$. The surface obtained from $\widetilde{X}_{\tau}$ satisfies $\mathbb{C}\left(\widetilde{X}_{\tau}\right) \cong \widetilde{K}_{a}$ for some $a$ since $\widetilde{X}_{\tau}$ has an order six automorphism acting on the Weierstrass points by a permutation the conjugacy class $[3,3]$. The surface $\widetilde{Y}_{w}$ has an order six Deck transformation for the map depicted in Fig. A-2 and an involution covered by $z \mapsto-z+5 w+1$. 


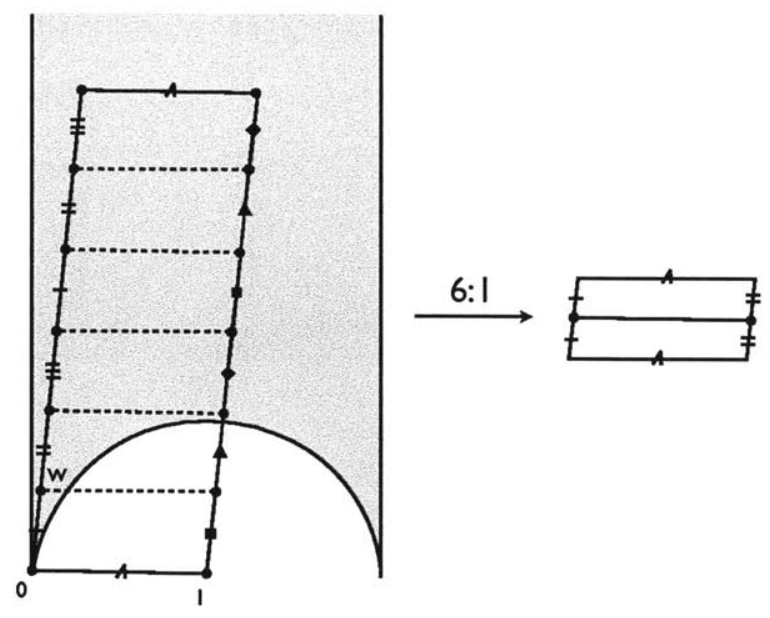

Figure A-2: For $w$ in the shaded domain $\tilde{V}$, the parallelogram $\widetilde{Q}_{w}$ has vertices $\{0,1,6 w, 6 w+1\}$. Gluing together the marked segments as indicated gives a genus two surface $\widetilde{Y}_{w}=Q_{w} / \sim$ with a degree six cyclic covering map to the double a parallelogram. The surface $\widetilde{Y}_{w}$ has an action of $D_{12}$ generated by the Deck transformation and the involution obtained by rotating $Q_{w}$ about $z=3 w+1 / 2$.

The isogeny $c_{\rho}: \operatorname{Jac}(X / r) \rightarrow \operatorname{Jac}(X / Z r)$ induced by the correspondence $X \rightarrow$ $X / r \times X / Z r$ is a degree three isogeny between elliptic curves and the map:

$$
\widetilde{\mathcal{M}}_{2}\left(D_{12}\right) \rightarrow X_{0}(3)
$$

given by $(X, \rho) \mapsto c_{\rho}$ is an isomorphism. The non-trivial outer automorphism of $D_{12}$ given by $\sigma(Z)=Z$ and $\sigma(r)=Z r$ induces the Atkin-Lehner involution on $\widetilde{\mathcal{M}}_{2}\left(D_{12}\right)$ and the unique nodal $D_{12}$-surface corresponds to the point of order three on $X_{0}(3)$. The function relating $\tau$ and $a$ is modular and the function relating $\tau$ and $w$ is a Riemann mapping between $\widetilde{U}$ and $\widetilde{V}$.

The surface obtained from $H_{\tau}$ has complex multiplication and is labeled by an orbifold point on a Hilbert modular surface iff $\tau$ is imaginary quadratic. When $\tau$ is in $\mathbb{Q}\left(\zeta_{3}\right)$, the vertices of $H_{\tau}$ lie in a lattice and the Teichmüller geodesic generated by the $Z$-eigenform for $\widetilde{X}_{\tau}$ is a Teichmüller curve associated to a square tiled surface. When $\tau$ is imaginary quadratic but not in $\mathbb{Q}\left(\zeta_{3}\right)$, the complex geodesic in $\mathcal{M}_{2}$ generated by the $Z$-eigenform on $\widetilde{X}_{\tau}$ is an example of the type studied in [Mc2] and has infinitely 
generated fundamental group. 


\section{A.2 Homeomorphism type of $W_{D}$}

\begin{tabular}{|c|c|c|c|c|}
\hline$D$ & $g\left(W_{D}\right)$ & $e_{2}\left(W_{D}\right)$ & $C\left(W_{D}\right)$ & $\chi\left(W_{D}\right)$ \\
\hline 5 & 0 & 1 & 1 & $-\frac{3}{10}$ \\
\hline 8 & 0 & 0 & 2 & $-\frac{3}{4}$ \\
\hline 9 & 0 & 1 & 2 & $-\frac{1}{2}$ \\
\hline 12 & 0 & 1 & 3 & $-\frac{3}{2}$ \\
\hline 13 & 0 & 1 & 3 & $-\frac{3}{2}$ \\
\hline 16 & 0 & 1 & 3 & $-\frac{3}{2}$ \\
\hline 17 & $\{0,0\}$ & $\{1,1\}$ & $\{3,3\}$ & $\left\{-\frac{3}{2},-\frac{3}{2}\right\}$ \\
\hline 20 & 0 & 0 & 5 & -3 \\
\hline 21 & 0 & 2 & 4 & -3 \\
\hline 24 & 0 & 1 & 6 & $-\frac{9}{2}$ \\
\hline 25 & $\{0,0\}$ & $\{0,1\}$ & $\{5,3\}$ & $\left\{-3,-\frac{3}{2}\right\}$ \\
\hline 28 & 0 & 2 & 7 & -6 \\
\hline 29 & 0 & 3 & 5 & $-\frac{9}{2}$ \\
\hline 32 & 0 & 2 & 7 & -6 \\
\hline 33 & $\{0,0\}$ & $\{1,1\}$ & $\{6,6\}$ & $\left\{-\frac{9}{2},-\frac{9}{2}\right\}$ \\
\hline 36 & 0 & 0 & 8 & -6 \\
\hline 37 & 0 & 1 & 9 & $-\frac{15}{2}$ \\
\hline 40 & 0 & 1 & 12 & $-\frac{21}{2}$ \\
\hline 41 & $\{0,0\}$ & $\{2,2\}$ & $\{7,7\}$ & $\{-6,-6\}$ \\
\hline 44 & 1 & 3 & 9 & $-\frac{21}{2}$ \\
\hline 45 & 1 & 2 & 8 & -9 \\
\hline 48 & 1 & 2 & 11 & -12 \\
\hline 49 & $\{0,0\}$ & $\{2,0\}$ & $\{10,8\}$ & $\{-9,-6\}$ \\
\hline
\end{tabular}

\begin{tabular}{|c|c|c|c|c|}
\hline$D$ & $g\left(W_{D}\right)$ & $e_{2}\left(W_{D}\right)$ & $C\left(W_{D}\right)$ & $\chi\left(W_{D}\right)$ \\
\hline 52 & 1 & 0 & 15 & -15 \\
\hline 53 & 2 & 3 & 7 & $-\frac{21}{2}$ \\
\hline 56 & 3 & 2 & 10 & -15 \\
\hline 57 & $\{1,1\}$ & $\{1,1\}$ & $\{10,10\}$ & $\left\{-\frac{21}{2},-\frac{21}{2}\right\}$ \\
\hline 60 & 3 & 4 & 12 & -18 \\
\hline 61 & 2 & 3 & 13 & $-\frac{33}{2}$ \\
\hline 64 & 1 & 2 & 17 & -18 \\
\hline 65 & $\{1,1\}$ & $\{2,2\}$ & $\{11,11\}$ & $\{-12,-12\}$ \\
\hline 68 & 3 & 0 & 14 & -18 \\
\hline 69 & 4 & 4 & 10 & -18 \\
\hline 72 & 4 & 1 & 16 & $-\frac{45}{2}$ \\
\hline 73 & $\{1,1\}$ & $\{1,1\}$ & $\{16,16\}$ & $\left\{-\frac{33}{2},-\frac{33}{2}\right\}$ \\
\hline 76 & 4 & 3 & 21 & $-\frac{57}{2}$ \\
\hline 77 & 5 & 4 & 8 & -18 \\
\hline 80 & 4 & 4 & 16 & -24 \\
\hline 81 & $\{2,0\}$ & $\{0,3\}$ & $\{16,14\}$ & $\left\{-18,-\frac{27}{2}\right\}$ \\
\hline 84 & 7 & 0 & 18 & -30 \\
\hline 85 & 6 & 2 & 16 & -27 \\
\hline 88 & 7 & 1 & 22 & $-\frac{69}{2}$ \\
\hline 89 & $\{3,3\}$ & $\{3,3\}$ & $\{14,14\}$ & $\left\{-\frac{39}{2},-\frac{39}{2}\right\}$ \\
\hline 92 & 8 & 6 & 13 & -30 \\
\hline 93 & 8 & 2 & 12 & -27 \\
\hline 96 & 8 & 4 & 20 & -36 \\
\hline
\end{tabular}

Table A.1: The Weierstrass curve $W_{D}$ is a finite volume hyperbolic orbifold and for $D>8$ its homeomorphism type is determined by the genus $g\left(W_{D}\right)$, the number of orbifold points of order two $e_{2}\left(W_{D}\right)$, the number of cusps $C\left(W_{D}\right)$ and the Euler characteristic $\chi\left(W_{D}\right)$. The values of these topological invariants are listed for each curve $W_{D}$ with $D<250$ as well as several larger discriminants. When $D>9$ with $D \equiv 1 \bmod 8$, the curve $W_{D}$ is reducible and the invariants are listed for both spin components with the invariants for $W_{D}^{0}$ appearing first. 


\begin{tabular}{|c|c|c|c|c|c|c|c|c|c|}
\hline$D$ & $g\left(W_{D}\right)$ & $e_{2}\left(W_{D}\right)$ & $C\left(W_{D}\right)$ & $\chi\left(W_{D}\right)$ & $D$ & $g\left(W_{D}\right)$ & $e_{2}\left(W_{D}\right)$ & $C\left(W_{D}\right)$ & $\chi\left(W_{D}\right)$ \\
\hline 97 & $\{4,4\}$ & $\{1,1\}$ & $\{19,19\}$ & $\left\{-\frac{51}{2},-\frac{51}{2}\right\}$ & 152 & 22 & 3 & 18 & $-\frac{123}{2}$ \\
\hline 100 & 4 & 0 & 30 & -36 & 153 & $\{10,10\}$ & $\{2,2\}$ & $\{26,26\}$ & $\{-45,-45\}$ \\
\hline 101 & 6 & 7 & 15 & $-\frac{57}{2}$ & 156 & 25 & 8 & 26 & -78 \\
\hline 104 & 9 & 3 & 20 & $-\frac{75}{2}$ & 157 & 20 & 3 & 25 & $-\frac{129}{2}$ \\
\hline 105 & $\{6,6\}$ & $\{2,2\}$ & $\{16,16\}$ & $\{-27,-27\}$ & 160 & 22 & 4 & 40 & -84 \\
\hline 108 & 10 & 3 & 21 & $-\frac{81}{2}$ & 161 & $\{14,14\}$ & $\{4,4\}$ & $\{20,20\}$ & $\{-48,-48\}$ \\
\hline 109 & 8 & 3 & 25 & $-\frac{81}{2}$ & 164 & 20 & 0 & 34 & -72 \\
\hline 112 & 10 & 2 & 29 & -48 & 165 & 24 & 4 & 18 & -66 \\
\hline 113 & $\{6,6\}$ & $\{2,2\}$ & $\{16,16\}$ & $\{-27,-27\}$ & 168 & 29 & 2 & 24 & -81 \\
\hline 116 & 11 & 0 & 25 & -45 & 169 & $\{14,7\}$ & $\{0,3\}$ & $\{37,39\}$ & $\left\{-63,-\frac{105}{2}\right\}$ \\
\hline 117 & 10 & 4 & 16 & -36 & 172 & 29 & 3 & 37 & $-\frac{189}{2}$ \\
\hline 120 & 16 & 2 & 20 & -51 & 173 & 22 & 7 & 13 & $-\frac{117}{2}$ \\
\hline 121 & $\{6,3\}$ & $\{3,0\}$ & $\{26,26\}$ & $\left\{-\frac{75}{2},-30\right\}$ & 176 & 27 & 6 & 29 & -84 \\
\hline 124 & 15 & 6 & 29 & -60 & 177 & $\{17,17\}$ & $\{1,1\}$ & $\{26,26\}$ & $\left\{-\frac{117}{2},-\frac{117}{2}\right\}$ \\
\hline 125 & 11 & 5 & 15 & $-\frac{75}{2}$ & 180 & 28 & 0 & 36 & -90 \\
\hline 128 & 13 & 4 & 22 & -48 & 181 & 26 & 5 & 33 & $-\frac{171}{2}$ \\
\hline 129 & $\{8,8\}$ & $\{3,3\}$ & $\{22,22\}$ & $\left\{-\frac{75}{2},-\frac{75}{2}\right\}$ & 184 & 37 & 2 & 38 & -111 \\
\hline 132 & 15 & 0 & 26 & -54 & 185 & $\{17,17\}$ & $\{4,4\}$ & $\{23,23\}$ & $\{-57,-57\}$ \\
\hline 133 & 15 & 2 & 22 & -51 & 188 & 31 & 10 & 19 & -84 \\
\hline 136 & 17 & 2 & 36 & -69 & 189 & 27 & 6 & 26 & -81 \\
\hline 137 & $\{9,9\}$ & $\{2,2\}$ & $\{19,19\}$ & $\{-36,-36\}$ & 192 & 31 & 4 & 34 & -96 \\
\hline 140 & 19 & 6 & 18 & -57 & 193 & $\{19,19\}$ & $\{1,1\}$ & $\{37,37\}$ & $\left\{-\frac{147}{2},-\frac{147}{2}\right\}$ \\
\hline 141 & 18 & 4 & 18 & -54 & 196 & 25 & 0 & 60 & -108 \\
\hline 144 & 11 & 4 & 38 & -60 & 197 & 26 & 5 & 21 & $-\frac{147}{2}$ \\
\hline 145 & $\{10,10\}$ & $\{2,2\}$ & $\{29,29\}$ & $\{-48,-48\}$ & 200 & 31 & 3 & 36 & $-\frac{195}{2}$ \\
\hline 148 & 20 & 0 & 37 & -75 & 201 & $\{20,20\}$ & $\{3,3\}$ & $\{34,34\}$ & $\left\{-\frac{147}{2},-\frac{147}{2}\right\}$ \\
\hline 149 & 16 & 7 & 19 & $-\frac{105}{2}$ & 204 & 38 & 6 & 40 & -117 \\
\hline
\end{tabular}




\begin{tabular}{|c|c|c|c|c|}
\hline$D$ & $g\left(W_{D}\right)$ & $e_{2}\left(W_{D}\right)$ & $C\left(W_{D}\right)$ & $\chi\left(W_{D}\right)$ \\
\hline 213 & 36 & 4 & 18 & -90 \\
\hline 216 & 38 & 3 & 46 & -2432 \\
\hline 217 & $\{25,25\}$ & $\{2,2\}$ & $\{38,38\}$ & $\{-87,-87\}$ \\
\hline 220 & 46 & 8 & 44 & -138 \\
\hline 221 & 32 & 8 & 30 & -96 \\
\hline 224 & 42 & 8 & 34 & -120 \\
\hline 225 & $\{21,16\}$ & $\{4,0\}$ & $\{42,42\}$ & $\{-84,-72\}$ \\
\hline 228 & 43 & 0 & 42 & -126 \\
\hline 229 & 42 & 5 & 37 & $-\frac{243}{2}$ \\
\hline 232 & 49 & 1 & 52 & $-\frac{297}{2}$ \\
\hline 233 & $\{27,27\}$ & $\{3,3\}$ & $\{26,26\}$ & $\left\{-\frac{159}{2},-\frac{159}{2}\right\}$ \\
\hline 236 & 45 & 9 & 35 & $-\frac{255}{2}$ \\
\hline 237 & 42 & 6 & 20 & -105 \\
\hline 240 & 52 & 4 & 40 & -144 \\
\hline 241 & $\{31,31\}$ & $\{3,3\}$ & $\{45,45\}$ & $\left\{-\frac{213}{2},-\frac{213}{2}\right\}$ \\
\hline 244 & 53 & 0 & 61 & -165 \\
\hline 245 & 40 & 6 & 24 & -105 \\
\hline 248 & 52 & 4 & 22 & -126 \\
\hline 249 & $\{32,32\}$ & $\{3,3\}$ & $\{40,40\}$ & $\left\{-\frac{207}{2},-\frac{207}{2}\right\}$ \\
\hline 41376 & 164821 & 112 & 1552 & -331248 \\
\hline 41377 & $\{113276,113276\}$ & $\{28,28\}$ & $\{1442,1442\}$ & $\{-228006,-228006\}$ \\
\hline 41380 & 178100 & 0 & 3154 & -359352 \\
\hline 41381 & 119380 & 89 & 665 & $-\frac{478935}{2}$ \\
\hline 41384 & 145957 & 68 & 884 & -292830 \\
\hline 41385 & $\{107869,107869\}$ & $\{24,24\}$ & $\{1284,1284\}$ & $\{-217032,-217032\}$ \\
\hline 41388 & 155386 & 54 & 1188 & -311985 \\
\hline 41389 & 146346 & 81 & 1475 & $-\frac{588411}{2}$ \\
\hline 41392 & 173203 & 48 & 2340 & -348768 \\
\hline
\end{tabular}




\section{Bibliography}

[At] M. F. Atiyah. Riemann surfaces and spin structures. Ann. scient. Éc. Norm. Sup. 4(1971), 47-62.

[Ba] M. Bainbridge. Euler characteristics of Teichmüller curves in genus two. Geom. Topol. 11(2007), 1887-2073.

[BaM] M. Bainbridge and M. Möller. Deligne-Mumford compactification of the real multiplication locus and Teichmüller curves in genus three. Preprint, arXiv:0911.4677.

[BL] C. Birkenhake and H. Lange. Complex Abelian Varieties, volume 302 of Grundlehren der Mathematischen Wissenschaften. Springer-Verlag, Berlin, 2004.

[BM1] I. Bouw and M. Möller. Differential equations associated with nonarithmetic Fuchsian groups. J. Lond. Math. Soc. 81(2010), 65-90.

[BM2] I. Bouw and M. Möller. Teichmüller curves, triangle groups, and Lyapunov exponents. Ann. of Math. 172(2010), 139-185.

[Bru] J. H. Bruinier. Hilbert modular forms and their applications. In The 1-2-3 of Modular Forms, Universitext, pages 105-179. Springer, Berlin, 2008.

[Ca] K. Calta. Veech surfaces and complete periodicity in genus two. J. Amer. Math. Soc. 17(2004), 871-908.

[Coh] H. Cohen. A Course in Computational Algebraic Number Theory. SpringerVerlag, Berlin, 2000.

[DS] F. Diamond and J. Shurman. A First Course in Modular Forms. Springer, 2005.

[KY] B. Klingler and A. Yafaev. The André-Oort conjecture. Preprint.

[KZ] M. Kontsevich and A. Zorich. Connected components of the moduli spaces of abelian differentials with prescribed singularities. Invent. Math. 153(2003), 631-678.

[Lo] P. Lochak. On arithmetic curves in the moduli space of curves. J. Inst. Math. Jussieu 4(2005), 443-508. 
[Ma] J. Manin. Parabolic points and zeta functions of modular curves. Izv. Akad. Nauk SSSR Ser. Math. 36(1972), 19-66.

[MT] H. Masur and S. Tabachnikov. Rational billiards and flat structures. In Handbook of dynamical systems, Vol. 1A, pages 1015-1089. North-Holland, Amsterdam, 2002.

[KMS] S. Kerckhoff, H. Masur and J. Smillie. Ergodicity of billiards flows and quadratic differentials. Ann. of Math. 124(1986), 293-311.

[Mc1] C. McMullen. Billiards and Teichmüller curves on Hilbert modular surfaces. J. Amer. Math. Soc. 16(2003), 857-885.

[Mc2] C. McMullen. Teichmüller geodesics of infinite complexity. Acta Math. 191(2003), 191-223.

[Mc3] C. McMullen. Teichmüller curves in genus two: Discriminant and spin. Math. Ann. 333(2005), 87-130.

[Mc4] C. McMullen. Dynamics of $\mathrm{SL}_{2}(\mathbb{R})$ over moduli space in genus two. Ann. of Math. 165(2007), 397-456.

[Mc5] C. McMullen. Teichmüller curves in genus two: Torsion divisors and ratios of sines. Invent. Math. 165, 651-672.

[Mo] M. Möller. Shimura and Teichmüller curves. Preprint, arxiv:math/0501333 (2010).

[Pr] A. Prestel. Die elliptischen Fixpunkte der Hilbertschen modulgruppen. Math. Ann. 177(1968), 181-209.

[Sh1] G. Shimura. On analytic families of polarized abelian varieties and automorphic functions. Ann. of Math. 78(1963), 149-192.

[Sh2] G. Shimura. Abelian Varieties with Complex Multiplication and Modular Functions, volume 46 of Princeton Mathematical Series. Princeton University Press, Princeton, NJ, 1998.

[Si] R. Silhol. Genus 2 translation surfaces with an order 4 automorphism. In The geometry of Riemann surfaces and abelian varieties, volume 397 of Contemp. Math., pages 207-213. Amer. Math. Soc., Providence, RI, 2006.

[Ti] J. Tits. Le Monstre. Astérisque (1985), 105-122. Seminar Bourbaki, Vol. $1983 / 84$.

[vdG] G. van der Geer. Hilbert Modular Surfaces, volume 16 of Ergebnisse der Mathematik und ihrer Grenzgebiete. Springer-Verlag, Berlin, 1988.

[Ve] W. A. Veech. Teichmüller curves in moduli space, Eisenstein series and an application to triangular billiards. Invent. Math. 97(1989), 553-583. 
[Zo] A. Zorich. Flat surfaces. In Frontiers in Number Theory, Physics and Geometry. Volume 1: On random matrices, zeta functions and dynamical systems, pages 439-586. Springer-Verlag, Berlin, 2006. 